\title{
A variational multiscale method for steady natural convection problem based on two-grid discretization
}

\author{
Xuesi Ma ${ }^{1,2^{*}}$, ZhenZhen $\mathrm{TaO}^{2}$ and Tong Zhang ${ }^{2,3}$
}

"Correspondence:

maxuesi@hpu.edu.cn

${ }^{1}$ Center for Intelligence Science and

Technology, School of Computer

Science, Beijing University of Posts

and Telecommunications, Beijing,

100876, P.R. China

${ }^{2}$ School of Mathematics \&

Information Science, Henan

Polytechnic University, Jiaozuo,

454003, P.R. China

Full list of author information is

available at the end of the article

\begin{abstract}
In this paper, we propose and analyze a two-grid variational multiscale method for the steady natural convection problem based on two local Gauss integrations technique. This method possesses the best algorithmic characteristics of both variational multiscale method and two-grid discretization. The main idea is to first solve the nonlinear steady natural convection problem on the coarse grid, then to use the coarse grid solution to fix the nonlinear terms, and to solve a linear problem on the fine grid. Stability and optimal error estimates of the discrete solutions in both one-grid and two-grid variational multiscale formulations are established. Finally, some numerical examples are presented to verify the method's promise and testify the theoretical predictions.
\end{abstract}

Keywords: natural convection problem; two-grid discretization; Oseen iteration; variational multiscale method; error estimates

\section{Introduction}

The natural convection phenomenon is present in many real situations such as room ventilation, double glass window design, etc. More importantly, it is behind the oceanic and atmospheric dynamics. Let $\Omega \subset \mathbb{R}^{d}(d=2$ or 3 ) be a regular bounded open domain, we consider the following steady natural convection problem:

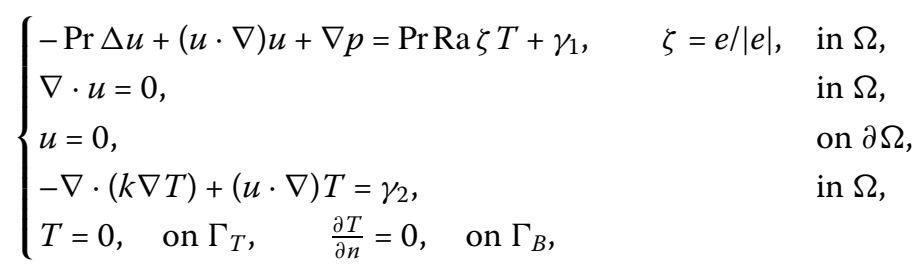

where $\Omega$ is a bounded convex polyhedral domain, the unknown functions are the velocity $u$, the pressure $p$, and the temperature T. $e$ is a unit vector in the direction of gravitational acceleration, $\gamma_{1}$ and $\gamma_{2}$ are known functions. $n$ is the outward unit normal to $\Omega$, and $\Gamma_{T}=\partial \Omega \backslash \Gamma_{B}$ where $\Gamma_{B}$ is a regular open subset of $\partial \Omega$. Pr, Ra, and $k>0$ denote Prandtl number, Rayleigh number, and thermal conductivity parameter, respectively.

The natural convection problem (1.1) is an important system with dissipative nonlinear terms in atmospheric dynamics. This system not only contains the velocity and pressure,

(c) $2016 \mathrm{Ma}$ et al. This article is distributed under the terms of the Creative Commons Attribution 4.0 International License (http://creativecommons.org/licenses/by/4.0/), which permits unrestricted use, distribution, and reproduction in any medium, provided you give appropriate credit to the original author(s) and the source, provide a link to the Creative Commons license, and indicate if changes were made. 
but also includes the temperature field, finding the numerical solution of problem (1.1) becomes a difficult task. For the research of the natural convection problem (1.1), there have enormous works been devoted to the development of efficient schemes ([1-9] and the references therein). For example, [3, 4] developed the mixed finite element method (FEM) for problem (1.1). Cibik and Kaya [5] constructed a projection-based stabilization finite element method. Galvin et al. [6] studied problem (1.1) with poor mass conservation in mixed finite element algorithm for flow problems of large rotation-free forcing in the momentum equations. Zhang et al. [7] developed the decoupled two-grid method. For the timedependent case, Benítez and Bermúdez [9] considered a second order Lagrange-Galerkin method. Boland and Layton [1] presented stability and error estimates for the Galerkin finite element spatial semi-discretization case. Manzari [8] used standard Galerkin FEM in spatial discretization and an explicit multistage Runge-Kutta scheme in the time domain for convection heat transfer problem.

It is well known that the small viscous problem is a challenge subject due to the singularity of the numerical solutions. As a result, much attention has been appealed to the recent years. There are several numerical schemes have been developed for the simulation of small viscous flows, such as the decoupled method [7], the variational multiscale method [10-18], the defect-correction method $[19,20]$ and so on. Among them, the variational multiscale method is a popular numerical technique. This method is on the base of the decomposition of the flow scales and definition of the large scales that is projected into appropriate subspaces. In this work, we propose a new variational multiscale method by defining the stabilization terms via two local Gauss integrations based on projection operators. A significant feature of this new variational multiscale method is that the stabilization terms are defined by the difference between a consistent and an under-integrated matrix only involving the velocity gradient (and temperature gradient), rather than the projection operator as used in the common variational multiscale method. This new variational multiscale method does not need to introduce extra variables and can keep good accuracy. Zhang et al. have made some numerical examples in [21] to verify the efficiency of this new variational multiscale method for problem (1.1), Shang [22] has presented an error analysis of two level subgrid stabilized Oseen iterative method for time-dependent Navier-Stokes equations. The main contribution of this work can be listed as follows. (1) The theoretical analysis of new variational multiscale method based on two local Gauss integrations for steady natural convection problem is provided. (2) A two-grid variational multiscale Oseen iterative scheme for the steady natural convection problem is developed, the corresponding error estimates are presented. Therefore, our work can be considered as an extension and supplement of the existing results $[18,21,22]$.

The paper is organized as follows. In Section 2, we present the notations and mathematical preliminaries for the considered problem and finite element spaces. Based on the elliptic projections of velocity and temperature, we formulate one-grid variational multiscale method, and apply the Oseen iterative scheme to linearize this nonlinear problem in Section 3. Section 4 develops and analyzes a two-grid variational multiscale Oseen iterative method. Finally, some numerical tests are presented to testify the correctness of the theoretical predictions and verify the effectiveness and efficiency of the developed method. 


\section{Preliminaries}

\subsection{Mathematical setting and basic results}

The function spaces for the velocity $u$, the pressure $p$, and the temperature $T$ are defined, respectively, by

$$
\begin{aligned}
& X=H_{0}^{1}(\Omega)^{d}=\left\{v \in H^{1}(\Omega)^{d}: v=0 \text { on } \partial \Omega\right\}, \\
& M=L_{0}^{2}(\Omega)=\left\{p \in L^{2}(\Omega):(p, 1)_{\Omega}=0\right\}, \\
& W=\left\{S \in H^{1}(\Omega): S=0 \text { on } \Gamma_{B}\right\}, \\
& V=H_{0, \mathrm{div}}^{1}(\Omega)=\{v \in X: \nabla \cdot v=0 \text { in } \Omega\},
\end{aligned}
$$

where the spaces $L^{2}(\Omega)^{m}, m=1,2,3$ are equipped with the standard $L^{2}$-scalar product $(\cdot, \cdot)$ and $L^{2}$-norm $\|\cdot\|_{0}, H^{j}(\Omega)$ denotes the standard Sobolev space with norm $\|\cdot\|_{j}$. The space $X$ is endowed with the usual scalar product $(\nabla u, \nabla v)$ and the norm $\|\nabla u\|_{0}$.

The weak form of (1.1) reads as follows: Find $(u, p, T) \in X \times M \times W$ such that

$$
\left\{\begin{array}{l}
\operatorname{Pr} a(u, v)+c(u, u, v)+b(v, p)-b(u, q)=\operatorname{Pr} \operatorname{Ra}(T \zeta, v)+\left(\gamma_{1}, v\right) \\
k \bar{a}(T, S)+\bar{c}(u, T, S)=\left(\gamma_{2}, S\right)
\end{array}\right.
$$

for all $(v, q, S) \in X \times M \times W$, where

$$
\begin{aligned}
& a(u, v)=(\nabla u, \nabla v), \quad \bar{a}(T, S)=(\nabla T, \nabla S), \quad b(v, q)=-(\nabla \cdot v, q), \\
& c(u, v, w)=((u \cdot \nabla) v, w)+\frac{1}{2}((\nabla \cdot u) v, w)=\frac{1}{2}((u \cdot \nabla) v, w)-\frac{1}{2}((u \cdot \nabla) w, v), \\
& \bar{c}(u, T, S)=((u \cdot \nabla) T, S)+\frac{1}{2}((\nabla \cdot u) T, S)=\frac{1}{2}((u \cdot \nabla) T, S)-\frac{1}{2}((u \cdot \nabla) S, T) .
\end{aligned}
$$

The following lemma and theorem provide some important results for bilinear terms $a(\cdot, \cdot), \bar{a}(\cdot, \cdot)$ and the existence and uniqueness of solutions for problem (1.1).

Lemma 2.1 (see [3]) For all $u, v, w \in X$ and $S, T \in W$, the following estimates hold.

$$
\begin{aligned}
& |a(u, v)| \leq\|\nabla u\|_{0}\|\nabla v\|_{0}, \quad|a(u, u)| \geq\|\nabla u\|_{0}^{2}, \\
& |\bar{a}(T, S)| \leq\|\nabla T\|_{0}\|\nabla S\|_{0}, \quad|\bar{a}(T, T)| \geq\|\nabla T\|_{0}^{2}, \\
& |c(u, v, w)| \leq N_{1}\|\nabla u\|_{0}\|\nabla v\|_{0}\|\nabla w\|_{0}, \quad|c(u, v, v)|=0, \\
& |\bar{c}(u, T, S)| \leq N_{2}\|\nabla u\|_{0}\|\nabla T\|_{0}\|\nabla S\|_{0}, \quad|\bar{c}(u, T, T)|=0,
\end{aligned}
$$

where $N_{1}=\sup _{0 \neq u, v, w \in X} \frac{|c(u, v, w)|}{\|\nabla u\|_{0}\|\nabla v\|_{0}\|\nabla w\|_{0}}$ and $N_{2}=\sup _{0 \neq u \in X, 0 \neq \theta, \psi \in W} \frac{|\bar{c}(u, \theta, \psi)|}{\|\nabla u\|_{0}\|\nabla \theta\|_{0}\|\nabla \nabla\|_{0}}$.

Theorem 2.2 (see [3]) Under the condition

$$
\mathrm{Ra}\left\|\gamma_{2}\right\|_{-1}+\frac{\operatorname{Pr}^{-1}}{k^{-1}+\operatorname{Pr} N_{2} k^{-2}}\left\|\gamma_{1}\right\|_{-1} \leq \frac{\operatorname{Pr}}{k^{-1}+\operatorname{Pr} N_{2} k^{-2}},
$$

problem (1.1) admits a unique solution $(u, T)$ and satisfies

$$
\|\nabla u\|_{0} \leq \operatorname{Pr}^{-1}\left\|\gamma_{1}\right\|_{-1}+\operatorname{Ra}^{-1}\left\|\gamma_{2}\right\|_{-1}, \quad\|\nabla T\|_{0} \leq k^{-1}\left\|\gamma_{2}\right\|_{-1} .
$$




\subsection{The finite element spaces}

To introduce the finite element discretization of (2.1), we assume $\mathcal{T}^{\mu}(\Omega)=\{K\}$ (here $\mu=H, h$ with $H \gg h$ ) to be a shape-regular triangulation of $\Omega$ into triangles or quadrilaterals (if $d=2$ ), or tetrahedrons or hexahedrons (if $d=3$ ) with mesh size $0<\mu<1$. The fine mesh $\mathcal{T}^{h}(\Omega)$ is found by a mesh refinement process of the coarse mesh. It is of worth to mention that it is not necessary for our method, nor needed for the results of our convergence theorems to hold. However, we assume the coarse and fine grids nested since it will simplify our analysis substantially. Let $X_{\mu} \subset X, M_{\mu} \subset M, W_{\mu} \subset W$ be three finite element spaces associated with $\mathcal{T}^{\mu}(\Omega)$ and satisfying the following assumptions.

(A1) Approximation. For each $(u, p, T) \in H^{k+1}(\Omega)^{d} \times H^{k}(\Omega) \times H^{k+1}(\Omega)$, there exists an approximation $\left(\pi_{\mu}^{1} u, \rho_{\mu} p, \pi_{\mu}^{2} T\right)$ such that

$$
\begin{aligned}
& \left\|\nabla\left(u-\pi_{\mu}^{1} u\right)\right\|_{0} \leq c \mu^{s}\|u\|_{1+s}, \quad\left\|p-\rho_{\mu} p\right\|_{0} \leq c u^{s}\|p\|_{s}, \\
& \left\|\nabla\left(T-\pi_{\mu}^{2} T\right)\right\|_{0} \leq c \mu^{s}\|T\|_{1+s}, \quad 0 \leq s \leq k,
\end{aligned}
$$

here and below, the letters $c$ and $C$ denote the general positive constants dependent at most on the coefficients of the equations and the domain, but independent of the mesh size and the iterative times $m$, furthermore, they are different at their different occurrences.

(A2) inf-sup condition. For the finite element spaces $X_{\mu}$ and $M_{\mu}$, there exists a constant $\beta>0$ such that

$$
\beta\left\|q_{\mu}\right\|_{0} \leq \sup _{v_{\mu} \in X_{\mu}, v_{\mu} \neq 0} \frac{\left(\nabla \cdot v_{\mu}, q_{\mu}\right)}{\left\|\nabla v_{\mu}\right\|_{0}}, \quad \forall q_{\mu} \in M_{\mu}
$$

It is noted that many mixed finite element pairs such as the Taylor-Hood element, the MINI element, and the $P_{2}-P_{0}$ element satisfy the above assumptions (A1) and (A2).

Set

$$
V_{\mu}=\left\{v_{\mu} \in X_{\mu}:\left(\nabla \cdot v_{\mu}, q_{\mu}\right)=0, \forall q_{\mu} \in M_{\mu}\right\} .
$$

It is well known that (see [10])

$$
\inf _{v_{\mu} \in V_{\mu}}\left\|\nabla\left(v-v_{\mu}\right)\right\|_{0} \leq C\left(1+\frac{1}{\beta}\right) \inf _{v_{\mu} \in X_{\mu}}\left\|\nabla\left(v-v_{\mu}\right)\right\|_{0}, \quad \forall v \in V .
$$

Under the above assumptions, the mixed finite element approximation of (2.1) reads: Find a pair $\left(u_{\mu}, p_{\mu}, T_{\mu}\right) \in X_{\mu} \times M_{\mu} \times W_{\mu}$ such that for all $\left(v_{\mu}, q_{\mu}, S_{\mu}\right)$

$$
\left\{\begin{array}{l}
\operatorname{Pr} a\left(u_{\mu}, v_{\mu}\right)+c\left(u_{\mu}, u_{\mu}, v_{\mu}\right)+b\left(v_{\mu}, p_{\mu}\right)-b\left(u_{\mu}, q_{\mu}\right)=\operatorname{Pr} \operatorname{Ra}\left(T_{\mu} \zeta, v_{\mu}\right)+\left(\gamma_{1}, v_{\mu}\right), \\
k \bar{a}\left(T_{\mu}, S_{\mu}\right)+\bar{c}\left(u_{\mu}, T_{\mu}, S_{\mu}\right)=\left(\gamma_{2}, S_{\mu}\right) .
\end{array}\right.
$$

The following theorem establishes the existence and uniqueness of the numerical solutions for problem (2.8) and provides the optimal $L^{2}$-norm error estimates.

Theorem 2.3 (see [7]) Under the assumptions of (A1), (A2), and some assumptions as regards the $k$, Pr, Ra, $\gamma_{1}, \gamma_{2}$, problem (2.8) possesses a unique solution $\left(u_{\mu}, p_{\mu}, T_{\mu}\right)$ and satisfies

$$
\left\|u-u_{\mu}\right\|_{0}+\left\|T-T_{\mu}\right\|_{0} \leq C \mu^{2}, \quad\left\|\nabla\left(u-u_{\mu}\right)\right\|_{0}+\left\|\nabla\left(T-T_{\mu}\right)\right\|_{0}+\left\|p-p_{\mu}\right\|_{0} \leq C \mu .
$$




\section{The variational multiscale method based on two local Gauss integrations}

In this section, we shall first formulate the variational multiscale method, and then develop the numerical scheme for the considered problem (2.1).

\subsection{The variational multiscale method}

Our variational multiscale method is based on two elliptic projection operators,

$$
\begin{aligned}
& \Pi_{\mu}^{1}: X \rightarrow R_{1}=\left\{v \in H_{0}^{1}(\Omega)^{d}:\left.v\right|_{K} \in\left(P_{1}\right)^{d}, \forall K \in E^{\mu}(\Omega)\right\}, \\
& \Pi_{\mu}^{2}: W \rightarrow R_{2}=\left\{S \in H_{0}^{1}(\Omega):\left.S\right|_{K} \in P_{1}, \forall K \in E^{\mu}(\Omega)\right\},
\end{aligned}
$$

which can be defined as follows (see [22]):

$$
\begin{aligned}
& \left(\nabla \Pi_{\mu}^{1} u, \nabla v\right)=(\nabla u, \nabla v), \quad \forall u \in X, v \in R_{1}, \\
& \left(\nabla \Pi_{\mu}^{2} T, \nabla S\right)=(\nabla T, \nabla S), \quad \forall T \in W, S \in R_{2},
\end{aligned}
$$

and have the following properties:

$$
\left\|\nabla \Pi_{\mu}^{1} u\right\|_{0} \leq\|\nabla u\|_{0}, \quad \forall u \in X, \quad\left\|\nabla \Pi_{\mu}^{2} T\right\|_{0} \leq\|\nabla T\|_{0}, \quad \forall T \in W
$$

where $R_{1}$ and $R_{2}$ are two spaces of polynomials of degree less than or equal to one.

Based on projections $\Pi_{\mu}^{1}$ and $\Pi_{\mu}^{2}$, we define the following stabilization terms:

$$
\begin{aligned}
& G_{1}(u, v)=\alpha_{1}\left(\nabla\left(I-\Pi_{\mu}^{1}\right) u, \nabla\left(I-\Pi_{\mu}^{1}\right) v\right), \quad \forall u, v \in X, \\
& G_{2}(T, S)=\alpha_{2}\left(\nabla\left(I-\Pi_{\mu}^{2}\right) T, \nabla\left(I-\Pi_{\mu}^{2}\right) S\right), \quad \forall T, S \in W,
\end{aligned}
$$

where $\alpha_{1}, \alpha_{2}>0$ are two user-defined stabilization parameters, typically chosen as $\alpha_{1}=$ $\alpha_{2}=\mathcal{O}(\mu)^{s}$ (here $s>0$ is a constant related to the finite elements used for the discretization of the considered problem).

Thanks to (3.2) and (3.3), the finite element variational multiscale method for problem (2.1) reads as follows: Find $\left(u_{\mu}, p_{\mu}, T_{\mu}\right) \in X_{\mu} \times M_{\mu} \times W_{\mu}$ such that

$$
\left\{\begin{array}{l}
\operatorname{Pr} a\left(u_{\mu}, v_{\mu}\right)+c\left(u_{\mu}, u_{\mu}, v_{\mu}\right)+b\left(v_{\mu}, p_{\mu}\right)-b\left(u_{\mu}, q_{\mu}\right)+G_{1}\left(u_{\mu}, v_{\mu}\right) \\
\quad=\operatorname{Pr} \operatorname{Ra}\left(T_{\mu} \zeta, v_{\mu}\right)+\left(\gamma_{1}, v_{\mu}\right) \\
k \bar{a}\left(T_{\mu}, S_{\mu}\right)+\bar{c}\left(u_{\mu}, T_{\mu}, S_{\mu}\right)+G_{2}\left(T_{\mu}, S_{\mu}\right)=\left(\gamma_{2}, S_{\mu}\right)
\end{array}\right.
$$

for all $\left(v_{\mu}, q_{\mu}, S_{\mu}\right) \in X_{\mu} \times M_{\mu} \times W_{\mu}$.

\subsection{Stability and convergence of scheme (3.4)}

The system (3.4) is nonlinear; it needs to be linearized in computations. A popular linearization process is the one based on the Newton iterative method. However, it is well known that the Newton iterative method is sensitive to the initial guess for the nonlinear iterations, i.e., to guarantee the convergence of the Newton iterations, the initial guess should be close enough to the solution $\left(u_{\mu}, T_{\mu}\right)$. Here we use the Oseen iterative method to solve (3.4).

From the definitions (3.2) and (3.3) of the projection operators $\Pi_{\mu}^{1}, \Pi_{\mu}^{2}$, we have

$$
\begin{aligned}
G_{1}(u, v) & =\alpha_{1}\left(\nabla\left(I-\Pi_{\mu}^{1}\right) u, \nabla\left(I-\Pi_{\mu}^{1}\right) v\right) \\
& =\alpha_{1}(\nabla u, \nabla v)-\alpha_{1}\left(\nabla \Pi_{\mu}^{1} u, \nabla v\right), \quad \forall u, v \in X,
\end{aligned}
$$




$$
\begin{aligned}
G_{2}(T, S) & =\alpha_{2}\left(\nabla\left(I-\Pi_{\mu}^{2}\right) T, \nabla\left(I-\Pi_{\mu}^{2}\right) S\right) \\
& =\alpha_{2}(\nabla T, \nabla S)-\alpha_{2}\left(\nabla \Pi_{\mu}^{2} T, \nabla S\right), \quad \forall T, S \in W .
\end{aligned}
$$

By applying the Oseen iterative method to the nonlinear problem (3.4) and thanks to (3.5) and (3.6), we develop the variational multiscale Oseen iterative scheme for the natural convection problem: Find an iterative solution $\left(u_{\mu}^{n}, p_{\mu}^{n}, T_{\mu}^{n}\right) \in X_{\mu} \times M_{\mu} \times W_{\mu}$ such that

$$
\left\{\begin{array}{l}
\left(\operatorname{Pr}+\alpha_{1}\right) a\left(u_{\mu}^{n}, v_{\mu}\right)+c\left(u_{\mu}^{n-1}, u_{\mu}^{n}, v_{\mu}\right)+b\left(v_{\mu}, p_{\mu}^{n}\right)-b\left(u_{\mu}^{n}, q_{\mu}\right) \\
\quad=\operatorname{Pr} \operatorname{Ra}\left(T_{\mu}^{n} \zeta, v_{\mu}\right)+\left(\gamma_{1}, v_{\mu}\right)+\alpha_{1}\left(\nabla \Pi_{\mu}^{1} u_{\mu}^{n-1}, \nabla v_{\mu}\right) \\
\left(k+\alpha_{2}\right) \bar{a}\left(T_{\mu}^{n}, S_{\mu}\right)+\bar{c}\left(u_{\mu}^{n-1}, T_{\mu}^{n}, S_{\mu}\right)=\left(\gamma_{2}, S_{\mu}\right)+\alpha_{2}\left(\nabla \Pi_{\mu}^{2} T_{\mu}^{n-1}, \nabla S_{\mu}\right)
\end{array}\right.
$$

for all $\left(v_{\mu}, q_{\mu}, S_{\mu}\right) \in X_{\mu} \times M_{\mu} \times W_{\mu}, n=1,2, \ldots$, with $u_{\mu}^{0}=0, T_{\mu}^{0}=0$.

Throughout this paper, we assume that $\mu$ is small enough such that the iterative scheme (3.7) is convergent. In order to keep notation brief, we define

$$
A=\operatorname{Pr}^{-1}\left\|\gamma_{1}\right\|_{-1}+\operatorname{Ra}^{-1}\left\|\gamma_{2}\right\|_{-1}, \quad B=k^{-1}\left\|\gamma_{2}\right\|_{-1} .
$$

Theorem 3.1 The iterative solution $\left(u_{\mu}^{n}, T_{\mu}^{n}\right)$ defined by (3.7) satisfies

$$
\left\|\nabla u_{\mu}^{n}\right\|_{0} \leq A, \quad\left\|\nabla T_{\mu}^{n}\right\|_{0} \leq B, \quad \forall n \geq 1
$$

Proof Let $S_{\mu}=T_{\mu}^{n}$ in the second equation of (3.7), we have

$$
\left(k+\alpha_{2}\right) \bar{a}\left(T_{\mu}^{n}, T_{\mu}^{n}\right)=\left(\gamma_{2}, T_{\mu}^{n}\right)+\alpha_{2}\left(\nabla \Pi_{\mu}^{2} T_{\mu}^{n-1}, \nabla T_{\mu}^{n}\right) .
$$

We use the Cauchy-Schwarz inequality and (3.1) to obtain

$$
\left(k+\alpha_{2}\right)\left\|\nabla T_{\mu}^{n}\right\|_{0} \leq\left\|\gamma_{2}\right\|_{-1}+\alpha_{2}\left\|\nabla \Pi_{\mu}^{2} T_{\mu}^{n-1}\right\|_{0} \leq\left\|\gamma_{2}\right\|_{-1}+\alpha_{2}\left\|\nabla T_{\mu}^{n-1}\right\|_{0}
$$

When $n=1$, we get

$$
\left(k+\alpha_{2}\right)\left\|\nabla T_{\mu}^{1}\right\|_{0} \leq\left\|\gamma_{2}\right\|_{-1} \quad \Rightarrow \quad\left\|\nabla T_{\mu}^{1}\right\|_{0} \leq \frac{1}{k+\alpha_{2}}\left\|\gamma_{2}\right\|_{-1} \leq k^{-1}\left\|\gamma_{2}\right\|_{-1},
$$

which shows that the second inequality of (3.8) holds for $n=1$. We now assume it holds for $n=J$ and prove it holds for $n=J+1$,

$$
\left(k+\alpha_{2}\right)\left\|\nabla T_{\mu}^{J+1}\right\|_{0} \leq\left\|\gamma_{2}\right\|_{-1}+\alpha_{2}\left\|\nabla T_{\mu}^{J}\right\|_{0} \leq k^{-1}\left(k+\alpha_{2}\right)\left\|\gamma_{2}\right\|_{-1}
$$

As a consequence one finds

$$
\left\|\nabla T_{\mu}^{J+1}\right\|_{0} \leq k^{-1}\left\|\gamma_{2}\right\|_{-1}
$$

Taking $v_{\mu}=u_{\mu}^{n}$ and $q_{\mu}=p_{\mu}^{n}$ in the first equation of (3.7), and using (3.1), we obtain

$$
\begin{aligned}
\left(\operatorname{Pr}+\alpha_{1}\right)\left\|\nabla u_{\mu}^{n}\right\|_{0} & \leq\left\|\gamma_{1}\right\|_{-1}+\alpha_{1}\left\|\nabla \Pi_{\mu}^{1} u_{\mu}^{n-1}\right\|_{0}+\operatorname{Pr} \operatorname{Ra}\left\|T_{\mu}^{n}\right\|_{-1} \\
& \leq\left\|\gamma_{1}\right\|_{-1}+\alpha_{1}\left\|\nabla u_{\mu}^{n-1}\right\|_{0}+\operatorname{Pr} \operatorname{Ra}^{-1}\left\|\gamma_{2}\right\|_{-1} .
\end{aligned}
$$


Due to the facts that $\alpha_{1}>0$ and $u_{\mu}^{0}=0$, we know that (3.8) holds for $n=1$. Assume that the first inequality of (3.8) holds for $n=J$. Taking $\left(v_{\mu}, q_{\mu}\right)=\left(u_{\mu}^{J+1}, p_{\mu}^{J+1}\right) \in X_{\mu} \times M_{\mu}$ in the first equation of (3.7) with $n=J+1$, we get

$$
\begin{aligned}
\left(\operatorname{Pr}+\alpha_{1}\right)\left\|\nabla u_{\mu}^{J+1}\right\|_{0} & \leq\left\|\gamma_{1}\right\|_{-1}+\alpha_{1}\left\|\nabla u_{\mu}^{J}\right\|_{0}+\operatorname{Pr} \operatorname{Ra}^{-1}\left\|\gamma_{2}\right\|_{-1} \\
& \leq\left\|\gamma_{1}\right\|_{-1}+\alpha_{1}\left(\operatorname{Pr}^{-1}\left\|\gamma_{1}\right\|_{-1}+\operatorname{Ra}^{-1}\left\|\gamma_{2}\right\|_{-1}\right)+\operatorname{Pr} \operatorname{Ra}^{-1}\left\|\gamma_{2}\right\|_{-1} \\
& \leq \frac{\operatorname{Pr}+\alpha_{1}}{\operatorname{Pr}}\left\|\gamma_{1}\right\|_{-1}+\left(\operatorname{Pr}+\alpha_{1}\right) \operatorname{Ra}^{-1}\left\|\gamma_{2}\right\|_{-1} .
\end{aligned}
$$

Consequently, we obtain

$$
\left\|\nabla u_{\mu}^{J+1}\right\|_{0} \leq \operatorname{Pr}^{-1}\left\|\gamma_{1}\right\|_{-1}+\operatorname{Ra}^{-1}\left\|\gamma_{2}\right\|_{-1}
$$

which with (3.9) completes the proof.

Theorem 3.2 Assume that $(u, T)$ is the nonsingular solution to the natural convection problem (1.1), $\alpha_{1}$ and $\alpha_{2}$ tend to zero as $\mu$ tends to zero. Under the condition of

$$
\operatorname{Pr}+2 \alpha_{1}-2 N_{1} A-2\left(k+\alpha_{2}\right)^{-1} \cdot \operatorname{Pr} \operatorname{Ra} N_{2} B>0,
$$

there exists $\mu_{0}>0$ such that for all $\mu \leq \mu_{0}$, the solution $\left(u_{\mu}^{n}, p_{\mu}^{n}, T_{\mu}^{n}\right)$ of (3.7) satisfies

$$
\begin{gathered}
\frac{1}{2} \operatorname{Pr}\left\|\nabla\left(u-u_{\mu}^{n}\right)\right\|_{0} \\
\leq C(\beta)\left(\frac{3}{2} \operatorname{Pr}+\alpha_{1}+2 N_{1} A+\frac{\operatorname{Pr} \operatorname{Ra} N_{2} B}{k+\alpha_{2}}\right) \inf _{\bar{u} \in X_{\mu}}\|\nabla(u-\bar{u})\|_{0} \\
+\sqrt{d} \inf _{\lambda_{\mu} \in M_{\mu}}\left\|p-\lambda_{\mu}\right\|_{0}+2 \alpha_{1} A+\left(N_{1} A+\frac{\operatorname{Pr} \operatorname{Ra} N_{2} B}{k+\alpha_{2}}\right)\left\|\nabla\left(u_{\mu}^{n}-u_{\mu}^{n-1}\right)\right\|_{0} \\
+\frac{\operatorname{Pr} \operatorname{Ra}\left(2 k+2 \alpha_{2}+N_{2} A\right)}{k+\alpha_{2}} \inf _{\bar{T} \in W_{\mu}}\|\nabla(T-\bar{T})\|_{0}+\frac{2 \operatorname{Pr} \operatorname{Ra} \alpha_{2} B}{k+\alpha_{2}}, \\
\left\|p-p_{\mu}^{n}\right\|_{0} \leq\left(\frac{\sqrt{d}}{\beta}+1\right) \inf _{\lambda_{\mu} \in M_{\mu}}\left\|p-\lambda_{\mu}\right\|_{0}+\frac{1}{\beta}\left(\operatorname{Pr}+\alpha_{1}+2 N_{1} A\right)\left\|\nabla\left(u-u_{\mu}^{n}\right)\right\|_{0} \\
+\frac{N_{1} A}{\beta}\left\|\nabla\left(u_{\mu}^{n}-u_{\mu}^{n-1}\right)\right\|_{0}+\frac{2 \alpha_{1} A}{\beta}+\frac{\operatorname{Pr} \operatorname{Ra}}{\beta}\left\|\nabla\left(T-T_{\mu}^{n}\right)\right\|_{0}, \\
\left\|\nabla\left(T-T_{\mu}^{n}\right)\right\|_{0} \leq \frac{2 k+2 \alpha_{2}+N_{2} A}{k+\alpha_{2}} \inf _{\bar{T} \in W_{\mu}}\|\nabla(T-\bar{T})\|_{0}+\frac{N_{2} B}{k+\alpha_{2}}\left\|\nabla\left(u-u_{\mu}^{n}\right)\right\|_{0} \\
+\frac{N_{2} B}{k+\alpha_{2}}\left\|\nabla\left(u_{\mu}^{n}-u_{\mu}^{n-1}\right)\right\|_{0}+\frac{2 \alpha_{2} B}{k+\alpha_{2}} .
\end{gathered}
$$

Here $\bar{u}, \lambda_{\mu}$, and $\bar{T}$ are the approximations of $u, p$, and $T$ in $X_{\mu}, M_{\mu}$, and $W_{\mu}$, respectively.

Remark 3.1 As the velocity $u$ and temperature $T$ are coupled in system (2.1), the error estimation for $T-T_{\mu}^{n}$ is coupled with the error $u-u_{\mu}^{n}$. 
Proof Setting $\left(e_{\mu}^{n}, \eta_{\mu}^{n}, \phi_{\mu}^{n}\right)=\left(u-u_{\mu}^{n}, p-p_{\mu}^{n}, T-T_{\mu}^{n}\right)$ and subtracting (3.7) from (2.1), we obtain the following error equations for the variational multiscale Oseen iterative scheme:

$$
\left\{\begin{array}{l}
\left(\operatorname{Pr}+\alpha_{1}\right)\left(\nabla e_{\mu}^{n}, \nabla v_{\mu}\right)+c\left(u, u, v_{\mu}\right)-c\left(u_{\mu}^{n-1}, u_{\mu}^{n}, v_{\mu}\right)+b\left(v_{\mu}, \eta_{\mu}^{n}\right)-b\left(e_{\mu}^{n}, q_{\mu}\right) \\
\quad=\operatorname{Pr} \operatorname{Ra}\left(\phi_{\mu}^{n} \zeta, v_{\mu}\right)+\alpha_{1}\left(\nabla u, \nabla v_{\mu}\right)-\alpha_{1}\left(\nabla \Pi_{\mu}^{1} u_{\mu}^{n-1}, \nabla v_{\mu}\right), \\
\left(k+\alpha_{2}\right) \bar{a}\left(\phi_{\mu}^{n}, S_{\mu}\right)+\bar{c}\left(u, T, S_{\mu}\right)-\bar{c}\left(u_{\mu}^{n-1}, T_{\mu}^{n}, S_{\mu}\right) \\
\quad=\alpha_{2}\left(\nabla T, \nabla S_{\mu}\right)-\alpha_{2}\left(\nabla \Pi_{\mu}^{2} T_{\mu}^{n-1}, \nabla S_{\mu}\right),
\end{array}\right.
$$

for all $\left(v_{\mu}, q_{\mu}, S_{\mu}\right) \in X_{\mu} \times M_{\mu} \times W_{\mu}$. Taking $v_{\mu} \in V_{\mu}$ and $q_{\mu}=0$, we get for any $\lambda_{\mu} \in M_{\mu}$

$$
\begin{aligned}
& \left(\operatorname{Pr}+\alpha_{1}\right)\left(\nabla e_{\mu}^{n}, \nabla v_{\mu}\right)+c\left(u, u, v_{\mu}\right)-c\left(u_{\mu}^{n-1}, u_{\mu}^{n}, v_{\mu}\right)-\left(\nabla \cdot v_{\mu}, p-\lambda_{\mu}\right) \\
& \quad=\operatorname{Pr} \operatorname{Ra}\left(\phi_{\mu}^{n} \zeta, v_{\mu}\right)+\alpha_{1}\left(\nabla u, \nabla v_{\mu}\right)-\alpha_{1}\left(\nabla \Pi_{\mu}^{1} u_{\mu}^{n-1}, \nabla v_{\mu}\right) .
\end{aligned}
$$

Splitting $e_{\mu}^{n}=\rho_{1}-\xi_{\mu}^{n}$ into $\rho_{1}=u-\bar{u}$ and $\xi_{\mu}^{n}=u_{\mu}^{n}-\bar{u}, \bar{u}$ is an approximation of $u$ in $V_{\mu}$, splitting $\phi_{\mu}^{n}=\rho_{2}-\varepsilon_{\mu}^{n}$ with $\rho_{2}=T-\bar{T}$ and $\varepsilon_{\mu}^{n}=T_{\mu}^{n}-\bar{T}, \bar{T}$ is an approximation of $T$ in $W_{\mu}$, noting that

$$
\begin{aligned}
c\left(u, u, v_{\mu}\right)-c\left(u_{\mu}^{n-1}, u_{\mu}^{n}, v_{\mu}\right) \\
=c\left(u, u-u_{\mu}^{n}+u_{\mu}^{n}, v_{\mu}\right)-c\left(u_{\mu}^{n-1}, u_{\mu}^{n}, v_{\mu}\right) \\
=c\left(u, e_{\mu}^{n}, v_{\mu}\right)+c\left(e_{\mu}^{n}, u_{\mu}^{n}, v_{\mu}\right)+c\left(u_{\mu}^{n}-u_{\mu}^{n-1}, u_{\mu}^{n}, v_{\mu}\right) \\
=c\left(u, \rho_{1}, v_{\mu}\right)-c\left(u, \xi_{\mu}^{n}, v_{\mu}\right)+c\left(\rho_{1}, u_{\mu}^{n}, v_{\mu}\right) \\
\quad-c\left(\xi_{\mu}^{n}, u_{\mu}^{n}, v_{\mu}\right)+c\left(u_{\mu}^{n}-u_{\mu}^{n-1}, u_{\mu}^{n}, v_{\mu}\right) .
\end{aligned}
$$

In the same way, we have

$$
\begin{aligned}
& \bar{c}\left(u, T, S_{\mu}\right)-\bar{c}\left(u_{\mu}^{n-1}, T_{\mu}^{n}, S_{\mu}\right) \\
& \quad=\bar{c}\left(u, \rho_{2}, S_{\mu}\right)-\bar{c}\left(u, \varepsilon_{\mu}^{n}, S_{\mu}\right)+\bar{c}\left(e_{\mu}^{n}, T_{\mu}^{n}, S_{\mu}\right)+\bar{c}\left(u_{\mu}^{n}-u_{\mu}^{n-1}, T_{\mu}^{n}, S_{\mu}\right) .
\end{aligned}
$$

From the second equation of (3.14), one finds

$$
\begin{aligned}
\left(k+\alpha_{2}\right)\left(\nabla \varepsilon_{\mu}^{n}, \nabla S_{\mu}\right)= & \left(k+\alpha_{2}\right)\left(\nabla \rho_{2}, \nabla S_{\mu}\right)+\bar{c}\left(u, T, S_{\mu}\right)-\bar{c}\left(u, T_{\mu}^{n}, S_{\mu}\right) \\
& +\alpha_{2}\left(\nabla \Pi_{\mu}^{2} T_{\mu}^{n-1}, \nabla S_{\mu}\right)-\alpha_{2}\left(\nabla T, \nabla S_{\mu}\right) .
\end{aligned}
$$

We take $S_{\mu}=\varepsilon_{\mu}^{n}$ using (3.17) to obtain

$$
\begin{aligned}
\left(k+\alpha_{2}\right)\left(\nabla \varepsilon_{\mu}^{n}, \nabla \varepsilon_{\mu}^{n}\right)= & \left(k+\alpha_{2}\right)\left(\nabla \rho_{2}, \nabla \varepsilon_{\mu}^{n}\right)+\bar{c}\left(u, \rho_{2}, \varepsilon_{\mu}^{n}\right)+\bar{c}\left(e_{\mu}^{n}, T_{\mu}^{n}, \varepsilon_{\mu}^{n}\right) \\
& +\bar{c}\left(u_{\mu}^{n}-u_{\mu}^{n-1}, T_{\mu}^{n}, \varepsilon_{\mu}^{n}\right)+\alpha_{2}\left(\nabla \Pi_{\mu}^{2} T_{\mu}^{n-1}, \nabla \varepsilon_{\mu}^{n}\right)-\alpha_{2}\left(\nabla T, \nabla \varepsilon_{\mu}^{n}\right) .
\end{aligned}
$$

Thanks to Theorem 2.2, (3.1) and Theorem 3.1 we derive that

$$
\begin{aligned}
\left(k+\alpha_{2}\right)\left\|\nabla \varepsilon_{\mu}^{n}\right\|_{0} \leq & \left(k+\alpha_{2}+N_{2} A\right)\left\|\nabla \rho_{2}\right\|_{0}+N_{2} B\left\|\nabla e_{\mu}^{n}\right\|_{0} \\
& +N_{2} B\left\|\nabla\left(u_{\mu}^{n}-u_{\mu}^{n-1}\right)\right\|_{0}+2 \alpha_{2} B .
\end{aligned}
$$


With the help of the triangle inequality one finds

$$
\begin{aligned}
\left(k+\alpha_{2}\right)\left\|\nabla \phi_{\mu}^{n}\right\|_{0} \leq & \left(2 k+2 \alpha_{2}+N_{2} A\right)\left\|\nabla \rho_{2}\right\|_{0}+N_{2} B\left\|\nabla e_{\mu}^{n}\right\|_{0} \\
& +N_{2} B\left\|\nabla\left(u_{\mu}^{n}-u_{\mu}^{n-1}\right)\right\|_{0}+2 \alpha_{2} B .
\end{aligned}
$$

We can get the error estimation (3.13) for $T-T_{\mu}^{n}$ by taking the infimum over $W_{\mu}$.

Now let us to prove (3.11). The applications of (3.15) and (3.16) lead to

$$
\begin{aligned}
\left(\operatorname{Pr}+\alpha_{1}\right)\left(\nabla \xi_{\mu}^{n}, \nabla v_{\mu}\right)= & \left(\operatorname{Pr}+\alpha_{1}\right)\left(\nabla \rho_{1}, \nabla v_{\mu}\right)+c\left(u, \rho_{1}, v_{\mu}\right)-c\left(u, \xi_{\mu}^{n}, v_{\mu}\right)+c\left(\rho_{1}, u_{\mu}^{n}, v_{\mu}\right) \\
& -c\left(\xi_{\mu}^{n}, u_{\mu}^{n}, v_{\mu}\right)+c\left(u_{\mu}^{n}-u_{\mu}^{n-1}, u_{\mu}^{n}, v_{\mu}\right)-\operatorname{Pr} \operatorname{Ra}\left(\phi_{\mu}^{n} \zeta, v_{\mu}\right) \\
& -\alpha_{1}\left(\nabla u, \nabla v_{\mu}\right)+\alpha_{1}\left(\nabla \Pi_{\mu}^{1} u_{\mu}^{n-1}, \nabla v_{\mu}\right)-\left(\nabla \cdot v_{\mu}, p-\lambda_{\mu}\right) .
\end{aligned}
$$

Taking $v_{\mu}=\xi_{\mu}^{n}$, we get

$$
\begin{aligned}
(\operatorname{Pr}+ & \left.\alpha_{1}\right)\left(\nabla \xi_{\mu}^{n}, \nabla \xi_{\mu}^{n}\right) \\
= & \left(\operatorname{Pr}+\alpha_{1}\right)\left(\nabla \rho_{1}, \nabla \xi_{\mu}^{n}\right)+c\left(u, \rho_{1}, \xi_{\mu}^{n}\right)+c\left(\rho_{1}, u_{\mu}^{n}, \xi_{\mu}^{n}\right)-c\left(\xi_{\mu}^{n}, u_{\mu}^{n}, \xi_{\mu}^{n}\right) \\
& +c\left(u_{\mu}^{n}-u_{\mu}^{n-1}, u_{\mu}^{n}, \xi_{\mu}^{n}\right)-\operatorname{Pr} \operatorname{Ra}\left(\phi_{\mu}^{n} \zeta, \xi_{\mu}^{n}\right)-\alpha_{1}\left(\nabla u, \nabla \xi_{\mu}^{n}\right) \\
& +\alpha_{1}\left(\nabla \Pi_{\mu}^{1} u_{\mu}^{n-1}, \nabla \xi_{\mu}^{n}\right)-\left(\nabla \cdot \xi_{\mu}^{n}, p-\lambda_{\mu}\right) .
\end{aligned}
$$

Thus

$$
\begin{aligned}
\left(\operatorname{Pr}+\alpha_{1}\right)\left\|\nabla \xi_{\mu}^{n}\right\|_{0} & \\
\leq & \left(\operatorname{Pr}+\alpha_{1}+N_{1}\|\nabla u\|_{0}+N_{1}\left\|\nabla u_{\mu}^{n}\right\|_{0}\right)\left\|\nabla \rho_{1}\right\|_{0}+N_{1}\left\|\nabla u_{\mu}^{n}\right\|_{0}\left\|\nabla\left(u_{\mu}^{n}-u_{\mu}^{n-1}\right)\right\|_{0} \\
& +\operatorname{Pr} \operatorname{Ra}\left\|\nabla \phi_{\mu}^{n}\right\|_{0}+\alpha_{1}\|\nabla u\|_{0}+\alpha_{1}\left\|\nabla \Pi_{\mu}^{1} u_{\mu}^{n-1}\right\|_{0} \\
& +N_{1}\left\|\nabla u_{\mu}^{n}\right\|_{0}\left\|\nabla \xi_{\mu}^{n}\right\|_{0}+\sqrt{d}\left\|p-\lambda_{\mu}\right\|_{0} .
\end{aligned}
$$

Making use of Theorem 2.2 and Theorem 3.1, we obtain

$$
\begin{aligned}
\left(\operatorname{Pr}+\alpha_{1}\right)\left\|\nabla \xi_{\mu}^{n}\right\|_{0} \leq & \left(\operatorname{Pr}+\alpha_{1}+2 N_{1} A\right)\left\|\nabla \rho_{1}\right\|_{0}+N_{1} A\left\|\nabla\left(u_{\mu}^{n}-u_{\mu}^{n-1}\right)\right\|_{0} \\
& +\operatorname{Pr} \operatorname{Ra}\left\|\nabla \phi_{\mu}^{n}\right\|_{0}+2 \alpha_{1} A+N_{1} A\left\|\nabla \xi_{\mu}^{n}\right\|_{0}+\sqrt{d}\left\|p-\lambda_{\mu}\right\|_{0} .
\end{aligned}
$$

To complete the proof, the bound (3.13) is inserted into the one of (3.18), this gives

$$
\begin{aligned}
& \left(\operatorname{Pr}+\alpha_{1}\right)\left\|\nabla \xi_{\mu}^{n}\right\|_{0} \\
& \leq\left(\operatorname{Pr}+\alpha_{1}+2 N_{1} A\right)\left\|\nabla \rho_{1}\right\|_{0}+N_{1} A\left\|\nabla\left(u_{\mu}^{n}-u_{\mu}^{n-1}\right)\right\|_{0}+2 \alpha_{1} A+N_{1} A\left\|\nabla \xi_{\mu}^{n}\right\|_{0} \\
& \quad+\sqrt{d}\left\|p-\lambda_{\mu}\right\|_{0}+\operatorname{Pr} \operatorname{Ra}\left[\frac{2 k+2 \alpha_{2}+N_{2} A}{k+\alpha_{2}} \inf _{\bar{T} \in W_{\mu}}\|\nabla(T-\bar{T})\|_{0}\right. \\
& \left.\quad+\frac{N_{2} B}{k+\alpha_{2}}\left\|\nabla\left(u-u_{\mu}^{n}\right)\right\|_{0}+\frac{N_{2} B}{k+\alpha_{2}}\left\|\nabla\left(u_{\mu}^{n}-u_{\mu}^{n-1}\right)\right\|_{0}+\frac{2 \alpha_{2} B}{k+\alpha_{2}}\right]
\end{aligned}
$$


thus

$$
\begin{aligned}
& \left(\operatorname{Pr}+\alpha_{1}-N_{1} A-\frac{\operatorname{Pr} \operatorname{Ra} N_{2} B}{k+\alpha_{2}}\right)\left\|\nabla \xi_{\mu}^{n}\right\|_{0} \\
& \leq\left(\operatorname{Pr}+\alpha_{1}+2 N_{1} A+\frac{\operatorname{Pr} \operatorname{Ra} N_{2} B}{k+\alpha_{2}}\right)\left\|\nabla \rho_{1}\right\|_{0}+\left(N_{1} A+\frac{\operatorname{Pr} \operatorname{Ra} N_{2} B}{k+\alpha_{2}}\right)\left\|\nabla\left(u_{\mu}^{n}-u_{\mu}^{n-1}\right)\right\|_{0} \\
& \quad+2 \alpha_{1} A+\sqrt{d}\left\|p-\lambda_{\mu}\right\|_{0} \\
& \quad+\frac{\operatorname{Pr} \operatorname{Ra}\left(2 k+2 \alpha_{2}+N_{2} A\right)}{k+\alpha_{2}} \inf _{\bar{T} \in W_{\mu}}\|\nabla(T-\bar{T})\|_{0}+\frac{2 \operatorname{Pr} \operatorname{Ra} \alpha_{2} B}{k+\alpha_{2}}
\end{aligned}
$$

Under the condition of (3.10) we find that

$$
\begin{aligned}
\frac{1}{2} \operatorname{Pr}\left\|\nabla e_{\mu}^{n}\right\|_{0} \leq & \frac{1}{2} \operatorname{Pr}\left(\left\|\nabla \rho_{1}\right\|_{0}+\left\|\nabla \xi_{\mu}^{n}\right\|_{0}\right) \\
\leq & \left(\frac{3}{2} \operatorname{Pr}+\alpha_{1}+2 N_{1} A+\frac{\operatorname{Pr} \operatorname{Ra} N_{2} B}{k+\alpha_{2}}\right)\left\|\nabla \rho_{1}\right\|_{0}+\sqrt{d}\left\|p-\lambda_{\mu}\right\|_{0} \\
& +2 \alpha_{1} A+\frac{2 \operatorname{Pr} \operatorname{Ra} \alpha_{2} B}{k+\alpha_{2}}+\left(N_{1} A+\frac{\operatorname{Pr} \operatorname{Ra} N_{2} B}{k+\alpha_{2}}\right)\left\|\nabla\left(u_{\mu}^{n}-u_{\mu}^{n-1}\right)\right\|_{0} \\
& +\frac{\operatorname{Pr} \operatorname{Ra}\left(2 k+2 \alpha_{2}+N_{2} A\right)}{k+\alpha_{2}} \inf _{\bar{T} \in W_{\mu}}\|\nabla(T-\bar{T})\|_{0} .
\end{aligned}
$$

Taking the infimum over $\bar{u} \in V_{\mu}, \lambda_{\mu} \in M_{\mu}$, and using (2.7), we get the required result (3.11).

Now, we present the convergence of $p-p_{\mu}^{n}$. Letting $\lambda_{\mu}$ be an approximation of $p$ in $M_{\mu}$ and setting $p-p_{\mu}^{n}=\left(p-\lambda_{\mu}\right)-\left(p_{\mu}^{n}-\lambda_{\mu}\right)$, from the first equation of (3.14) and (3.16) we get

$$
\begin{aligned}
(\nabla & \left.\cdot v_{\mu}, p_{\mu}^{n}-\lambda_{\mu}\right) \\
= & \left(\nabla \cdot v_{\mu}, p-\lambda_{\mu}\right)-\left(\operatorname{Pr}+\alpha_{1}\right)\left(\nabla e_{\mu}^{n}, \nabla v_{\mu}\right)-c\left(u, e_{\mu}^{n}, v_{\mu}\right)-c\left(e_{\mu}^{n}, u_{\mu}^{n}, u_{\mu}\right) \\
& -c\left(u_{\mu}^{n}-u_{\mu}^{n-1}, u_{\mu}^{n}, v_{\mu}\right)+\alpha_{1}\left(\nabla u, \nabla v_{\mu}\right)-\alpha_{1}\left(\nabla \Pi_{\mu}^{1} u_{\mu}^{n-1}, \nabla v_{\mu}\right)+\operatorname{Pr} \operatorname{Ra}\left(\phi_{\mu}^{n} \zeta, v_{\mu}\right) \\
\leq & {\left[\sqrt{d}\left\|p-\lambda_{\mu}\right\|_{0}+\left(\operatorname{Pr}+\alpha_{1}+N_{1}\|\nabla u\|_{0}+N_{1}\left\|\nabla u_{\mu}^{n}\right\|_{0}\right)\left\|\nabla e_{\mu}^{n}\right\|_{0}+\operatorname{Pr} \operatorname{Ra}\left\|\nabla \phi_{\mu}^{n}\right\|_{0}\right.} \\
& \left.+N_{1}\left\|\nabla u_{\mu}^{n}\right\|_{0}\left\|\nabla\left(u_{\mu}^{n}-u_{\mu}^{n-1}\right)\right\|_{0}+\alpha_{1}\left(\|\nabla u\|_{0}+\left\|\nabla u_{\mu}^{n-1}\right\|_{0}\right)\right]\left\|\nabla v_{\mu}\right\|_{0},
\end{aligned}
$$

which together with the inf-sup condition and the bounds of $\|\nabla u\|_{0},\left\|\nabla u_{\mu}^{n-1}\right\|_{0},\left\|\nabla u_{\mu}^{n}\right\|_{0}$ yields

$$
\begin{aligned}
& \beta\left\|p_{\mu}^{n}-\lambda_{\mu}\right\|_{0} \\
& \leq \sqrt{d}\left\|p-\lambda_{\mu}\right\|_{0}+\left(\operatorname{Pr}+\alpha_{1}+N_{1}\|\nabla u\|_{0}+N_{1}\left\|\nabla u_{\mu}^{n}\right\|_{0}\right)\left\|\nabla e_{\mu}^{n}\right\|_{0} \\
&+N_{1}\left\|\nabla u_{\mu}^{n}\right\|_{0}\left\|\nabla\left(u_{\mu}^{n}-u_{\mu}^{n-1}\right)\right\|_{0}+\alpha_{1}\left(\|\nabla u\|_{0}+\left\|\nabla u_{\mu}^{n-1}\right\|_{0}\right)+\operatorname{Pr} \operatorname{Ra}\left\|\nabla \phi_{\mu}^{n}\right\|_{0} \\
& \leq \sqrt{d}\left\|p-\lambda_{\mu}\right\|_{0}+\left(\operatorname{Pr}+\alpha_{1}+2 N_{1} A\right)\left\|\nabla\left(u-u_{\mu}^{n}\right)\right\|_{0} \\
&+N_{1} A\left\|\nabla\left(u_{\mu}^{n}-u_{\mu}^{n-1}\right)\right\|_{0}+2 \alpha_{1} A+\operatorname{Pr} \operatorname{Ra}\left\|\nabla\left(T-T_{\mu}^{n}\right)\right\|_{0} .
\end{aligned}
$$

Using the triangle inequality and taking the infimum over $\lambda_{\mu} \in M_{\mu}$, we finish the proof. 
Corollary 3.2 Under the condition of Theorem 3.2, the solution $\left(u_{\mu}^{n}, p_{\mu}^{n}, T_{\mu}^{n}\right) \in X_{\mu} \times M_{\mu} \times$ $W_{\mu}$ defined by (3.7) satisfies

$$
\begin{gathered}
\left\|\nabla\left(u-u_{\mu}^{n}\right)\right\|_{0}+\left\|p-p_{\mu}^{n}\right\|_{0}+\left\|\nabla\left(T-T_{\mu}^{n}\right)\right\|_{0} \\
\leq c \mu^{s}+C_{1} \alpha_{1}+C_{2} \alpha_{2}+C_{3}\left\|\nabla\left(u_{\mu}^{n}-u_{\mu}^{n-1}\right)\right\|_{0} .
\end{gathered}
$$

Remark 3.3 Corollary 3.2 shows that to ensure a good approximation, the variational multiscale Oseen iterations should converge sufficiently. Moreover, the estimator suggests the choice of the stabilization parameters $\alpha_{1}=\mathcal{O}\left(\mu^{s}\right)$ and $\alpha_{2}=\mathcal{O}\left(\mu^{s}\right)$ which ensure optimal convergence.

\section{Two-grid variational multiscale method}

Combining two-grid discretization strategy with the variational multiscale Oseen iterative method presented in the previous section, we develop the two-grid variational multiscale Oseen iterative method as follows.

Step 1. Find a coarse grid iterative solution $\left(u_{H}^{m}, p_{H}^{m}, T_{H}^{m}\right) \in X_{H} \times M_{H} \times W_{H}$ such that

$$
\left\{\begin{array}{l}
\left(\operatorname{Pr}+\alpha_{1}\right) a\left(u_{H}^{m}, v_{H}\right)+c\left(u_{H}^{m-1}, u_{H}^{m}, v_{H}\right)+b\left(v_{H}, p_{H}^{m}\right)-b\left(u_{H}^{m}, q_{H}\right) \\
\quad=\operatorname{Pr} \operatorname{Ra}\left(T_{H}^{m} \zeta, v_{H}\right)+\left(\gamma_{1}, v_{H}\right)+\alpha_{1}\left(\nabla \Pi_{H}^{1} u_{H}^{m-1}, \nabla v_{H}\right), \\
\left(k+\alpha_{2}\right) \bar{a}\left(T_{H}^{m}, S_{H}\right)+\bar{c}\left(u_{H}^{m-1}, T_{H}^{m}, S_{H}\right)=\left(\gamma_{2}, S_{H}\right)+\alpha_{2}\left(\nabla \Pi_{H}^{2} T_{H}^{m-1}, \nabla S_{H}\right),
\end{array}\right.
$$

for all $\left(v_{H}, q_{H}, S_{H}\right) \in X_{H} \times M_{H} \times W_{H}, n=1,2, \ldots, m$, and $u_{H}^{0}=0, T_{H}^{0}=0$.

Step 2. Find a fine grid solution $\left(u_{m h}, p_{m h}, T_{m h}\right) \in X_{h} \times M_{h} \times W_{h}$ such that for all $\left(v_{h}, q_{h}, S_{h}\right) \in X_{h} \times M_{h} \times W_{h}$

$$
\left\{\begin{array}{l}
\operatorname{Pr} a\left(u_{m h}, v_{h}\right)+c\left(u_{H}^{m}, u_{m h}, v_{h}\right)+c\left(u_{m h}, u_{H}^{m}, v_{h}\right)+b\left(v_{h}, p_{m h}\right)-b\left(u_{m h}, q_{h}\right) \\
\quad=\operatorname{Pr} \operatorname{Ra}\left(T_{m h} \zeta, v_{h}\right)+\left(\gamma_{1}, v_{h}\right)+c\left(u_{H}^{m}, u_{H}^{m}, v_{h}\right), \\
k \bar{a}\left(T_{m h}, S_{h}\right)+\bar{c}\left(u_{H}^{m}, T_{m h}, S_{h}\right)+\bar{c}\left(u_{m h}, T_{H}^{m}, S_{h}\right)=\left(\gamma_{2}, S_{h}\right)+\bar{c}\left(u_{H}^{m}, T_{H}^{m}, S_{h}\right) .
\end{array}\right.
$$

Remark 4.1 In our two-grid method, stabilization is only employed for the coarse grid nonlinear problem, while the fine grid linear problem is a standard one-step Newton linearization. Therefore, Pr, Ra, $k$ should be in the range such that the standard linear problem on the fine grid is nonsingular and solvable.

Remark 4.2 The numerical algorithm (4.1) is a full linearization problem based on the Newton iterative scheme. Similarly, based on the Oseen iterative scheme, we can develop the following partial linearization problem: Find a fine grid solution $\left(u_{m h}, p_{m h}, T_{m h}\right) \in X_{h} \times$ $M_{h} \times W_{h}$ such that for all $\left(v_{h}, q_{h}, S_{h}\right) \in X_{h} \times M_{h} \times W_{h}$

$$
\left\{\begin{array}{l}
\operatorname{Pr} a\left(u_{m h}, v_{h}\right)+c\left(u_{H}^{m}, u_{m h}, v_{h}\right)+b\left(v_{h}, p_{m h}\right)-b\left(u_{m h}, q_{h}\right) \\
\quad=\operatorname{Pr} \operatorname{Ra}\left(T_{m h} \zeta, v_{h}\right)+\left(\gamma_{1}, v_{h}\right) \\
k \bar{a}\left(T_{m h}, S_{h}\right)+\bar{c}\left(u_{H}^{m}, T_{m h}, S_{h}\right)=\left(\gamma_{2}, S_{h}\right)
\end{array}\right.
$$

Theorem 4.1 Assume $\left(\tilde{u}, \lambda_{h}, \tilde{T}\right)$ be an approximation of $(u, p, T)$ in $X_{h} \times M_{h} \times W_{h}$, under the conditions of Theorem 3.2 and $\frac{1}{2} \operatorname{Pr}-N_{1} A-k^{-1} \operatorname{Pr} \operatorname{Ra} N_{2} B>0$. The solution 
$\left(u_{m h}, p_{m h}, T_{m h}\right)$ defined by scheme (4.1) satisfies

$$
\begin{aligned}
\frac{1}{2} \operatorname{Pr} \| & \nabla\left(u-u_{m h}\right) \|_{0} \\
\leq & C(\beta)\left(\frac{3}{2} \operatorname{Pr}+2 N_{1} A+\operatorname{Pr} \operatorname{Ra}^{-1} N_{2} B\right) \inf _{\tilde{u} \in X_{h}}\|\nabla(u-\tilde{u})\|_{0} \\
& +\sqrt{d} \inf _{\lambda_{h} \in M_{h}}\left\|p-\lambda_{h}\right\|_{0} \\
& +\left(N_{1}\left\|\nabla\left(u-u_{H}^{m}\right)\right\|_{0}+\operatorname{Pr} \operatorname{Ra} k^{-1} N_{2}\left\|\nabla\left(T-T_{H}^{m}\right)\right\|_{0}\right)\left\|\nabla\left(u-u_{H}^{m}\right)\right\|_{0} \\
& +\operatorname{Pr} \operatorname{Ra} k^{-1}\left(2 k+N_{2} A+N_{2}\left\|\nabla\left(u-u_{H}^{m}\right)\right\|_{0}\right) \inf _{\tilde{T} \in W_{h}}\|\nabla(T-\tilde{T})\|_{0}, \\
\| p- & p_{m h}\left\|_{0} \leq\left(1+\frac{\sqrt{d}}{\beta}\right) \inf _{\lambda_{h} \in M_{h}}\right\| p-\lambda_{h}\left\|_{0}+\frac{1}{\beta}\left(\operatorname{Pr}+2 N_{1} A\right)\right\| \nabla\left(u-u_{m h}\right) \|_{0} \\
& +\frac{N_{1}}{\beta}\left\|\nabla\left(u-u_{H}^{m}\right)\right\|_{0}^{2}+\frac{\operatorname{Pr} \operatorname{Ra}}{\beta}\left\|\nabla\left(T-T_{m h}\right)\right\|_{0}, \\
\| \nabla( & \left.-T_{m h}\right) \|_{0} \\
\leq & k^{-1}\left(2 k+N_{2} A+N_{2}\left\|\nabla\left(u-u_{H}^{m}\right)\right\|_{0}\right) \inf _{\tilde{T} \in W_{h}}\|\nabla(T-\tilde{T})\|_{0} \\
& +k^{-1} N_{2} B\left\|\nabla\left(u-u_{m h}\right)\right\|_{0}+k^{-1} N_{2}\left\|\nabla\left(u-u_{H}^{m}\right)\right\|_{0}\left\|\nabla\left(T-T_{H}^{m}\right)\right\|_{0} .
\end{aligned}
$$

Proof Setting $\left(e_{m}, \eta_{m}, \phi_{m}\right)=\left(u-u_{m h}, p-p_{m h}, T-T_{m h}\right)$ and subtracting (4.1) from (2.1), for all $\left(v_{h}, q_{h}, S_{h}\right) \in X_{h} \times M_{h} \times W_{h}$ we have

$$
\left\{\begin{array}{l}
\operatorname{Pr}\left(\nabla e_{m}, \nabla v_{h}\right)+c\left(u, u, v_{h}\right)-c\left(u_{H}^{m}, u_{m h}, v_{h}\right)-c\left(u_{m h}, u_{H}^{m}, v_{h}\right)-\left(\nabla \cdot v_{h}, p-p_{m h}\right) \\
\quad+\left(\nabla \cdot e_{m}, q_{h}\right)=\operatorname{Pr} \operatorname{Ra}\left(\phi_{m} \zeta, v_{h}\right)-c\left(u_{H}^{m}, u_{H}^{m}, v_{h}\right), \\
k\left(\nabla \phi_{m}, \nabla S_{h}\right)+\bar{c}\left(u, T, S_{h}\right)-\bar{c}\left(u_{H}^{m}, T_{m h}, S_{h}\right)-\bar{c}\left(u_{m h}, T_{H}^{m}, S_{h}\right) \\
\quad=-\bar{c}\left(u_{H}^{m}, T_{H}^{m}, S_{h}\right) .
\end{array}\right.
$$

The remainder of the proof follows that of Theorem 3.2. Let $\left(\tilde{u}, \lambda_{h}, \tilde{T}\right)$ be an approximation of $(u, p, T)$. By limiting $v_{h} \in V_{h}$, splitting $e_{m}=\chi_{1}-\varphi_{h}$ with $\chi_{1}=u-\tilde{u}, \varphi_{h}=u_{m h}-\tilde{u}$, and splitting $\phi_{m}=\chi_{2}-\psi_{h}$ with $\chi_{2}=T-\tilde{T}, \psi_{h}=T_{m h}-\tilde{T}$, the trilinear terms transform into

$$
\begin{aligned}
& c\left(u, u, v_{h}\right)-c\left(u_{H}^{m}, u_{m h}, v_{h}\right)-c\left(u_{m h}, u_{H}^{m}, v_{h}\right)+c\left(u_{H}^{m}, u_{H}^{m}, v_{h}\right) \\
& \quad=c\left(\left(u-u_{H}^{m}\right)+u_{H}^{m}, u, v_{h}\right)-c\left(u_{H}^{m}, u_{m h}, v_{h}\right)+c\left(u-u_{m h}, u_{H}^{m}, v_{h}\right)-c\left(u-u_{H}^{m}, u_{H}^{m}, v_{h}\right) \\
& \quad=c\left(e_{m}, u_{H}^{m}, v_{h}\right)+c\left(u_{H}^{m}, e_{m}, v_{h}\right)+c\left(u-u_{H}^{m}, u-u_{H}^{m}, v_{h}\right) .
\end{aligned}
$$

In the same way, one finds

$$
\begin{aligned}
& \bar{c}\left(u, T, S_{h}\right)-\bar{c}\left(u_{H}^{m}, T_{m h}, S_{h}\right)-\bar{c}\left(u_{m h}, T_{H}^{m}, S_{h}\right)+\bar{c}\left(u_{H}^{m}, T_{H}^{m}, S_{h}\right) \\
& \quad=\bar{c}\left(u, \phi_{m}, S_{h}\right)-\bar{c}\left(u-u_{H}^{m}, \phi_{m}, S_{h}\right)+\bar{c}\left(e_{m}, T_{H}^{m}, S_{h}\right)+\bar{c}\left(u-u_{H}^{m}, T-T_{H}^{m}, S_{h}\right) .
\end{aligned}
$$


Inserting (4.7) and (4.8) into (4.6) we get

$$
\left\{\begin{array}{l}
\operatorname{Pr}\left(\nabla e_{m}, \nabla v_{h}\right)+c\left(e_{m}, u_{H}^{m}, v_{h}\right)+c\left(u_{H}^{m}, e_{m}, v_{h}\right)+c\left(u-u_{H}^{m}, u-u_{H}^{m}, v_{h}\right) \\
\quad-\left(\nabla \cdot v_{h}, p-p_{m h}\right)+\left(\nabla \cdot e_{m}, q_{h}\right)=\operatorname{Pr} \operatorname{Ra}\left(\phi_{m} \zeta, v_{h}\right), \\
k\left(\nabla \phi_{m}, \nabla S_{h}\right)+\bar{c}\left(u, \phi_{m}, S_{h}\right)-\bar{c}\left(u-u_{H}^{m}, \phi_{m}, S_{h}\right)+\bar{c}\left(e_{m}, T_{H}^{m}, S_{h}\right) \\
\quad+\bar{c}\left(u-u_{H}^{m}, T-T_{H}^{m}, S_{h}\right)=0 .
\end{array}\right.
$$

Setting $S_{h}=\psi_{h}$, the second equation of (4.9) can be written as

$$
\begin{aligned}
k\left(\nabla \psi_{h}, \nabla \psi_{h}\right) & k\left(\nabla \chi_{2}, \nabla \psi_{h}\right)+\bar{c}\left(u, \phi_{m}, \psi_{h}\right)-\bar{c}\left(u-u_{H}^{m}, \phi_{m}, \psi_{h}\right) \\
& +\bar{c}\left(e_{m}, T_{H}^{m}, \psi_{h}\right)+\bar{c}\left(u-u_{H}^{m}, T-T_{H}^{m}, \psi_{h}\right) \\
= & k\left(\nabla \chi_{2}, \nabla \psi_{h}\right)+\bar{c}\left(u, \chi_{2}, \psi_{h}\right)-\bar{c}\left(u-u_{H}^{m}, \chi_{2}, \psi_{h}\right) \\
& +\bar{c}\left(e_{m}, T_{H}^{m}, \psi_{h}\right)+\bar{c}\left(u-u_{H}^{m}, T-T_{H}^{m}, \psi_{h}\right),
\end{aligned}
$$

as a consequence we derive that

$$
\begin{aligned}
k\left\|\nabla \psi_{h}\right\|_{0} \leq & \left(k+N_{2}\|\nabla u\|_{0}+N_{2}\left\|\nabla\left(u-u_{H}^{m}\right)\right\|_{0}\right)\left\|\nabla \chi_{2}\right\|_{0}+N_{2}\left\|\nabla T_{H}^{m}\right\|_{0}\left\|\nabla e_{m}\right\|_{0} \\
& +N_{2}\left\|\nabla\left(u-u_{H}^{m}\right)\right\|_{0}\left\|\nabla\left(T-T_{H}^{m}\right)\right\|_{0} \\
\leq & \left(k+N_{2} A+N_{2}\left\|\nabla\left(u-u_{H}^{m}\right)\right\|_{0}\right)\left\|\nabla \chi_{2}\right\|_{0}+N_{2} B\left\|\nabla e_{m}\right\|_{0} \\
& +N_{2}\left\|\nabla\left(u-u_{H}^{m}\right)\right\|_{0}\left\|\nabla\left(T-T_{H}^{m}\right)\right\|_{0}
\end{aligned}
$$

Applying the triangle inequality, and taking the infimum over $W_{h}$, we obtain the proof (4.5).

Now, we present the error for $u-u_{m h}$. Setting $v_{h} \in V_{h}$ and $q_{h}=0$, the first equation of (4.6) can be written as

$$
\begin{aligned}
\operatorname{Pr}\left(\nabla \varphi_{h}, \nabla v_{h}\right)= & \operatorname{Pr}\left(\nabla \chi_{1}, \nabla v_{h}\right)+c\left(e_{m}, u_{H}^{m}, v_{h}\right)+c\left(u_{H}^{m}, e_{m}, v_{h}\right) \\
& +c\left(u-u_{H}^{m}, u-u_{H}^{m}, v_{h}\right)-\left(\nabla \cdot v_{h}, p-\lambda_{h}\right)-\operatorname{Pr} \operatorname{Ra}\left(\phi_{m} \zeta, v_{h}\right)
\end{aligned}
$$

Taking $v_{h}=\varphi_{h}$, which leads to

$$
\begin{aligned}
\operatorname{Pr}\left(\nabla \varphi_{h}, \nabla \varphi_{h}\right)= & \operatorname{Pr}\left(\nabla \chi_{1}, \nabla \varphi_{h}\right)+c\left(e_{m}, u_{H}^{m}, \varphi_{h}\right)+c\left(u_{H}^{m}, \chi_{1}, \varphi_{h}\right) \\
& +c\left(u-u_{H}^{m}, u-u_{H}^{m}, \varphi_{h}\right)-\left(\nabla \cdot \varphi_{h}, p-\lambda_{h}\right)-\operatorname{Pr} \operatorname{Ra}\left(\phi_{m} \zeta, \varphi_{h}\right) .
\end{aligned}
$$

Using Theorem 3.1 we obtain

$$
\begin{aligned}
\operatorname{Pr}\left\|\nabla \varphi_{h}\right\|_{0} \leq & \left(\operatorname{Pr}+2 N_{1}\left\|\nabla u_{H}^{m}\right\|_{0}\right)\left\|\nabla \chi_{1}\right\|_{0}+N_{1}\left\|\nabla\left(u-u_{H}^{m}\right)\right\|_{0}^{2} \\
& +\operatorname{Pr} \operatorname{Ra}\left\|\nabla \phi_{m}\right\|_{0}+\sqrt{d}\left\|p-\lambda_{h}\right\|_{0}+N_{1}\left\|\nabla u_{H}^{m}\right\|_{0}\left\|\nabla \varphi_{h}\right\|_{0} \\
\leq & \left(\operatorname{Pr}+2 N_{1} A\right)\left\|\nabla \chi_{1}\right\|_{0}+N_{1}\left\|\nabla\left(u-u_{H}^{m}\right)\right\|_{0}^{2} \\
& +\operatorname{Pr} \operatorname{Ra}\left\|\nabla \phi_{m}\right\|_{0}+\sqrt{d}\left\|p-\lambda_{h}\right\|_{0}+N_{1} A\left\|\nabla \varphi_{h}\right\|_{0} .
\end{aligned}
$$


Inserting (4.5) into (4.11) to derive that

$$
\begin{aligned}
\operatorname{Pr}\left\|\nabla \varphi_{h}\right\|_{0} \leq & \left(\operatorname{Pr}+2 N_{1} A\right)\left\|\nabla \chi_{1}\right\|_{0}+N_{1}\left\|\nabla\left(u-u_{H}^{m}\right)\right\|_{0}^{2}+\sqrt{d}\left\|_{p}-\lambda_{h}\right\|_{0}+N_{1} A\left\|\nabla \varphi_{h}\right\|_{0} \\
& +\operatorname{Pr} \operatorname{Ra}\left[k^{-1}\left(2 k+N_{2} A+N_{2}\left\|\nabla\left(u-u_{H}^{m}\right)\right\|_{0}\right) \underset{\tilde{T} \in W_{h}}{\inf }\|\nabla(T-\tilde{T})\|_{0}\right. \\
& \left.+k^{-1} N_{2} B\left\|\nabla\left(u-u_{m h}\right)\right\|_{0}+k^{-1} N_{2}\left\|\nabla\left(u-u_{H}^{m}\right)\right\|_{0}\left\|\nabla\left(T-T_{H}^{m}\right)\right\|_{0}\right] \\
\leq & \left(\operatorname{Pr}+2 N_{1} A+k^{-1} \operatorname{Pr} \operatorname{Ra} N_{2} B\right)\left\|\nabla \chi_{1}\right\|_{0}+N_{1}\left\|\nabla\left(u-u_{H}^{m}\right)\right\|_{0}^{2}+\sqrt{d}\left\|p-\lambda_{h}\right\|_{0} \\
& +\left(N_{1} A+k^{-1} \operatorname{Pr} \operatorname{Ra} N_{2} B\right)\left\|\nabla \varphi_{h}\right\|_{0} \\
& +k^{-1} \operatorname{Pr} \operatorname{Ra} N_{2}\left\|\nabla\left(u-u_{H}^{m}\right)\right\|_{0}\left\|\nabla\left(T-T_{H}^{m}\right)\right\|_{0} \\
& +k^{-1} \operatorname{Pr} \operatorname{Ra}\left(2 k+N_{2} A+N_{2}\left\|\nabla\left(u-u_{H}^{m}\right)\right\|_{0}\right) \underset{\tilde{T} \in W_{h}}{\inf }\|\nabla(T-\tilde{T})\|_{0} .
\end{aligned}
$$

Under the assumption of Theorem 4.1 one finds

$$
\begin{aligned}
\frac{1}{2} \operatorname{Pr}\left\|\nabla \varphi_{h}\right\|_{0} \leq & \left(\operatorname{Pr}+2 N_{1} A+k^{-1} \operatorname{Pr} \operatorname{Ra} N_{2} B\right)\left\|\nabla \chi_{1}\right\|_{0}+N_{1}\left\|\nabla\left(u-u_{H}^{m}\right)\right\|_{0}^{2} \\
& +\sqrt{d}\left\|p-\lambda_{h}\right\|_{0}+k^{-1} \operatorname{Pr} \operatorname{Ra} N_{2}\left\|\nabla\left(u-u_{H}^{m}\right)\right\|_{0}\left\|\nabla\left(T-T_{H}^{m}\right)\right\|_{0} \\
& +k^{-1} \operatorname{Pr} \operatorname{Ra}\left(2 k+N_{2} A+N_{2}\left\|\nabla\left(u-u_{H}^{m}\right)\right\|_{0}\right) \underset{\tilde{T} \in W_{h}}{\inf }\|\nabla(T-\tilde{T})\|_{0}
\end{aligned}
$$

Thanks to the triangle inequality and (2.7), and taking the infimum over $\left(\tilde{u}, \lambda_{h}\right) \in X_{h} \times M_{h}$, we finish the proof of (4.3).

Finally, we take $q_{h}=0$ in the first equation of (4.6) to derive that

$$
\begin{aligned}
(\nabla \cdot & \left.v_{h}, p_{m h}-\lambda_{h}\right) \\
= & \left(\nabla \cdot v_{h}, p-\lambda_{h}\right)-\operatorname{Pr}\left(\nabla e_{m}, \nabla v_{h}\right)-c\left(e_{m}, u_{H}^{m}, v_{h}\right)-c\left(u_{H}^{m}, e_{m}, v_{h}\right) \\
& -c\left(u-u_{H}^{m}, u-u_{H}^{m}, v_{h}\right)+\operatorname{Pr} \operatorname{Ra}\left(\phi_{m} \zeta, v_{h}\right) \\
\leq & {\left[\sqrt{d}\left\|p-\lambda_{h}\right\|_{0}+\left(\operatorname{Pr}+2 N_{1}\left\|\nabla u_{H}^{m}\right\|_{0}\right)\left\|\nabla e_{m}\right\|_{0}+N_{1}\left\|\nabla\left(u-u_{H}^{m}\right)\right\|_{0}^{2}\right.} \\
& \left.+\operatorname{Pr} \operatorname{Ra}\left\|\nabla \phi_{h}\right\|_{0}\right]\left\|\nabla v_{h}\right\|_{0},
\end{aligned}
$$

which combining (2.6) with Theorem 3.2 yields

$$
\begin{aligned}
\beta\left\|p_{m h}-\lambda_{h}\right\|_{0} \leq & \sqrt{d}\left\|p-\lambda_{h}\right\|_{0}+\left(\operatorname{Pr}+2 N_{1} A\right)\left\|\nabla e_{m}\right\|_{0} \\
& +N_{1}\left\|\nabla\left(u-u_{H}^{m}\right)\right\|_{0}^{2}+\operatorname{Pr} \operatorname{Ra}\left\|\nabla \phi_{h}\right\|_{0}
\end{aligned}
$$

by using $\left\|p-p_{m h}\right\|_{0} \leq\left\|p_{m h}-\lambda_{h}\right\|_{0}+\left\|p-\lambda_{h}\right\|_{0}$ we have

$$
\begin{aligned}
\left\|p-p_{m h}\right\|_{0} \leq & \left(1+\frac{\sqrt{d}}{\beta}\right)\left\|p-\lambda_{h}\right\|_{0}+\frac{1}{\beta}\left(\operatorname{Pr}+2 N_{1} A\right)\left\|\nabla\left(u-u_{m h}\right)\right\|_{0} \\
& +\frac{N_{1}}{\beta}\left\|\nabla\left(u-u_{H}^{m}\right)\right\|_{0}^{2}+\frac{\operatorname{Pr} \operatorname{Ra}}{\beta}\left\|\nabla\left(T-T_{m h}\right)\right\|_{0} .
\end{aligned}
$$

We derive the error estimation (4.5) by taking the infimum over $\lambda_{h} \in M_{h}$. 


\section{Numerical experiments}

In order to gain insights on the established theoretical results of one-grid and two-grid variational multiscale methods for the natural convection problem in Sections 3 and 4, we present two numerical examples in this section. Our main interests are to verify the orders of convergence and compare the performances of different numerical schemes. For comparison, the numerical results of the standard Galerkin FEM (2.8) are also provided. In all experiments, the steady natural convection problem is defined on a convex domain $\Omega=$ $[0,1] \times[0,1]$ and the variational multiscale parameters $\alpha_{1}=\alpha_{2}=0.1 h^{2}$. The mesh consists of triangular elements that are obtained by dividing $\Omega$ into subsquares of equal size and then drawing the diagonal in each sub-square. The software FreeFEM++, developed by Hecht et al. [23], is used in our experiments and the linear solver UMFPACK is adopted in our program. All numerical experiments reported in this paper were performed on a PC with a core process (i5-3210M) and 4GB of random access memory.

\subsection{An analytical solution: convergence validation}

In this test our purpose is to verify the theoretical results of Theorems 2.3, 3.2, and 4.1 which have been established in Sections 3 and 4, respectively. The model parameters Pr, $\mathrm{Ra}$, and $k$ are simply set to 1 , the functions $\gamma_{1}$ and $\gamma_{2}$ are given by the following exact solution:

$$
\left\{\begin{array}{l}
u_{1}=10 x^{2}(x-1)^{2} y(y-1)(2 y-1), \\
u_{2}=-10 x(x-1)(2 x-1) y^{2}(y-1)^{2}, \\
p=10(2 x-1)(2 y-1) \\
T=10 x^{2}(x-1)^{2} y(y-1)(2 y-1)-10 x(x-1)(2 x-1) y^{2}(y-1)^{2},
\end{array}\right.
$$

where the components of $u$ are denoted by $u_{1}$ and $u_{2}$ for convenience.

In order to show the prominent features of the two-grid variational multiscale Oseen iterative scheme (4.1), we compare the numerical results of one-grid variational multiscale scheme (3.7) and the standard Galerkin FEM (2.8). We consider the second order discretization, where the Taylor-Hood element is applied to approximate the velocity and pressure; the piecewise quadratic element is used to simulate the temperature. In this case, the orders of convergence of velocity in $H^{1}$ norm, pressure in $L^{2}$ norm, and temperature in $H^{1}$ norm should be 2 . In the following test, we will verify these orders of convergence. $\|\cdot\|_{0}$ denotes the $L^{2}$-norm and $\|\nabla \cdot\|_{0}$ is the $H^{1}$-semi-norm.

In Table 1, we show the relative errors of the standard Galerkin FEM (2.8) between the exact solution and the numerical approximations. As observed from Table 1, the contraction factors of errors $\frac{\left\|\nabla\left(u-u_{h}\right)\right\|_{0}}{\|\nabla u\|_{0}}, \frac{\left\|\nabla\left(T-T_{h}\right)\right\|_{0}}{\|\nabla T\|_{0}}$, and $\frac{\left\|p-p_{h}\right\|_{0}}{\|p\|_{0}}$ become smaller and smaller as the mesh is refined, and the orders of these relative errors are around $\frac{1}{4}$ as the mesh is refined once.

In contrast, we show the errors between the exact solution and the numerical solutions of both one-grid and two-grid variational multiscale Oseen iterative schemes (2.8) and (4.1) in Tables 2-3, respectively. We observe almost no differences when comparing the errors in Tables 2-3 with the same mesh sizes $h=\frac{1}{4}, \frac{1}{9}, \frac{1}{16}, \frac{1}{25}, \frac{1}{36}, \frac{1}{49}$ with $H=\sqrt{h}$. From these data we can see that the one-grid and two-grid variational multiscale schemes retain the same order of accuracy with second order discretization as the standard Galerkin FEM.

Finally, we compare the CPU time among different algorithms in Tables 1-3. From these tables, we can see that the two-grid variational multiscale Oseen iterative method (4.1) 
Table 1 The errors of standard Galerkin FEM (2.8) for the steady natural convection equations

\begin{tabular}{rlllllll}
\hline $\mathbf{1 / h}$ & $\frac{\left\|\nabla\left(\boldsymbol{u}-\boldsymbol{u}_{\boldsymbol{h}}\right)\right\|_{\mathbf{0}}}{\|\nabla \boldsymbol{u}\|_{\mathbf{0}}}$ & Rate & $\frac{\left\|\boldsymbol{p}-\boldsymbol{p}_{\boldsymbol{h}}\right\|_{\mathbf{0}}}{\|\boldsymbol{p}\|_{\mathbf{0}}}$ & Rate & $\frac{\left\|\nabla\left(\boldsymbol{T}-\boldsymbol{T}_{\boldsymbol{h}}\right)\right\|_{\mathbf{0}}}{\|\nabla \boldsymbol{T}\|_{\mathbf{0}}}$ & Rate & CPU(S) \\
\hline 4 & 0.166184 & - & 0.0485766 & - & 0.0962482 & - & 0.066 \\
9 & 0.0354715 & 1.90444 & 0.00956673 & 2.00369 & 0.0211698 & 1.86743 & 0.305 \\
16 & 0.0114207 & 1.96978 & 0.00302598 & 2.00057 & 0.00682614 & 1.96713 & 0.977 \\
25 & 0.00470301 & 1.98792 & 0.00123938 & 2.00012 & 0.0028114 & 1.98789 & 2.503 \\
36 & 0.00227273 & 1.99434 & 0.000597686 & 2.00003 & 0.00135839 & 1.99453 & 5.081 \\
49 & 0.00122789 & 1.99702 & 0.000322615 & 2.00001 & 0.000733861 & 1.99718 & 9.981 \\
\hline
\end{tabular}

Table 2 The errors of variational multiscale method (3.7) for the steady natural convection equations

\begin{tabular}{clllllll}
\hline $\mathbf{1} / \boldsymbol{h}$ & $\frac{\left\|\nabla\left(\boldsymbol{u}-\boldsymbol{u}_{\boldsymbol{h}}\right)\right\|_{\mathbf{0}}}{\|\nabla \boldsymbol{u}\|_{\mathbf{0}}}$ & Rate & $\frac{\left\|\boldsymbol{p}-\boldsymbol{p}_{\boldsymbol{h}}\right\|_{\mathbf{0}}}{\|\boldsymbol{p}\|_{\mathbf{0}}}$ & Rate & $\frac{\left\|\nabla\left(\boldsymbol{T}-\boldsymbol{T}_{\boldsymbol{h}}\right)\right\|_{\mathbf{0}}}{\|\nabla \boldsymbol{T}\|_{\mathbf{0}}}$ & Rate & CPU(S) \\
\hline 4 & 0.166197 & - & 0.0485818 & - & 0.0962482 & - & 0.08 \\
9 & 0.0354721 & 1.90451 & 0.009566804 & 2.0038 & 0.0211698 & 1.86743 & 0.283 \\
16 & 0.0114203 & 1.96981 & 0.00302599 & 2.00059 & 0.00682614 & 1.96713 & 0.901 \\
25 & 0.00470303 & 1.98793 & 0.00123938 & 2.00012 & 0.0028114 & 1.98789 & 2.207 \\
36 & 0.00227273 & 1.99434 & 0.000597686 & 2.00003 & 0.00135839 & 1.99453 & 4.682 \\
49 & 0.00122789 & 1.99703 & 0.000322615 & 2.00001 & 0.000733861 & 1.99718 & 8.804 \\
\hline
\end{tabular}

Table 3 The errors of two-grid variational multiscale method (4.1) for the steady natural convection equations

\begin{tabular}{lclllllll}
\hline $\mathbf{1 / H}$ & $\mathbf{1 / h}$ & $\frac{\left\|\nabla\left(\boldsymbol{u}-\boldsymbol{u}_{\boldsymbol{h}}\right)\right\|_{\mathbf{0}}}{\|\nabla \boldsymbol{u}\|_{\mathbf{0}}}$ & Rate & $\frac{\left\|\boldsymbol{p}-\boldsymbol{p}_{\boldsymbol{h}}\right\|_{\mathbf{0}}}{\|\boldsymbol{p}\|_{\mathbf{0}}}$ & Rate & $\frac{\left\|\nabla\left(\boldsymbol{T}-\boldsymbol{T}_{\boldsymbol{h}}\right)\right\|_{\mathbf{0}}}{\|\nabla \boldsymbol{V}\|_{\mathbf{0}}}$ & Rate & CPU(S) \\
\hline 2 & 4 & 0.166184 & - & 0.0485766 & - & 0.0962505 & - & 0.044 \\
3 & 9 & 0.0354715 & 1.90444 & 0.00956675 & 2.00369 & 0.0211715 & 1.86736 & 0.143 \\
4 & 16 & 0.0114202 & 1.96978 & 0.00302598 & 2.00057 & 0.00682667 & 1.96713 & 0.393 \\
5 & 25 & 0.00470301 & 1.98792 & 0.00123938 & 2.00012 & 0.00281136 & 1.98788 & 0.915 \\
6 & 36 & 0.00227273 & 1.99434 & 0.000597686 & 2.00003 & 0.0013585 & 1.99451 & 1.834 \\
7 & 49 & 0.00122789 & 1.99702 & 0.000322615 & 2.00001 & 0.000733929 & 1.99716 & 3.390 \\
\hline
\end{tabular}

uses less time than the numerical schemes (2.8) and (3.7). From the above tables, we can see that the two-grid variational multiscale Oseen iterative method shows a good performance for the steady natural convection equations due to this scheme not only keeping a good accuracy but also taking the least computational cost.

\subsection{Thermal driven cavity problem}

The problem of thermal driven cavity is used as a suitable benchmark for testing the natural convection problem in the literature. The simplicity of the geometry and the clear boundary conditions make this test attractive. The domain consists of a square cavity with differentially heated vertical walls where the right and left walls are kept at $T_{r}$ and $T_{l}$, respectively, with $T_{r}>T_{l}$. The remaining walls are insulated and there is no heat transfer through them. The boundary conditions are no-slip boundary conditions for the velocity at four walls $(u=0)$ and Dirichlet boundary conditions for the temperature at vertical walls. As the horizontal walls are adiabatic, we employ $\frac{\partial T}{\partial n}=0$. Figure 1 shows the physical domain of the thermal driven cavity flow problem. In this test, we follow the parameters set by Cibik and Kaya in [5] and take $k=1, b=0, T_{l}=0$, and $T_{r}=1$. While we consider the air as the cavity filling fluid in our model, we take the fixed value $\operatorname{Pr}=0.71$. We perform our computations for a Rayleigh number varying from $10^{3}$ to $10^{5}$. The performance of the two-grid variational multiscale method (4.1) is compared with the famous benchmark so- 


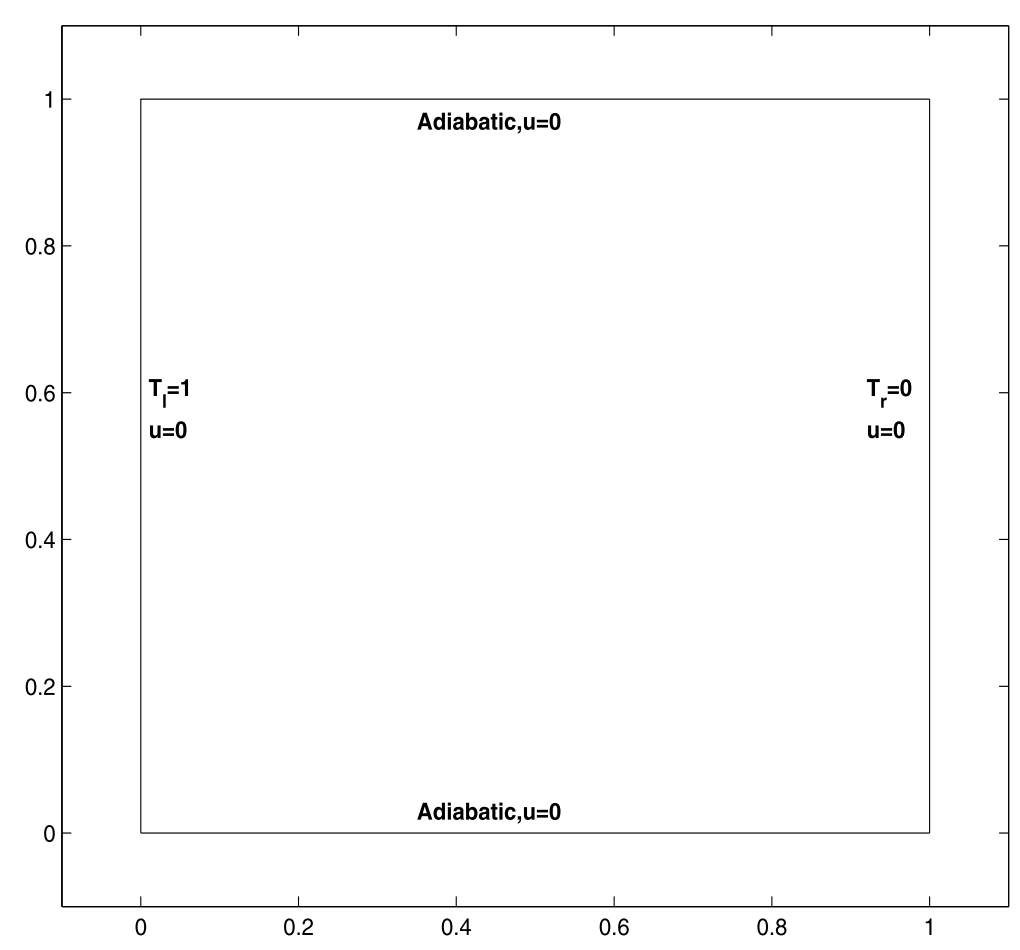

Figure 1 The physical domain with its boundary conditions.

lution of [24] and some other authors such as Cibik and Kaya [5], Manzari [8], and Wan et al. [25]. We also present the results of the standard Galekrkin FEM (2.8) where we keep the same mesh sizes for the two-grid variational multiscale method (4.1) and the one-grid variational multiscale method (3.7). Numerical simulations are obtained on the uniform grid $64 \times 64$. The mesh sizes of two-grid algorithms are $H=\frac{1}{8}$ and $h=\frac{1}{64}$.

We start our illustrations by giving peak values of the vertical velocity at $y=0.5$ and the horizontal velocity at $x=0.5$. Table 4 summarizes the maximum vertical velocity values at mid-height and at mid-width for different Rayleigh numbers. We use SGFEM, OGVMM, TGVMM to denote standard Galerkin FEM, one-grid, and two-grid variational multiscale methods, respectively. For quantitative assessment, we include those velocity values obtained by $[5,8,24,25]$. As can be observed, as the Rayleigh number takes values $10^{3}$ to $10^{5}$, the results of two-grid variational multiscale Oseen iterative method are in excellent agreement with the benchmark data even at coarser grid $H=\frac{1}{8}$.

Next, we present the vertical and horizontal velocity at the mid-height and mid-width in Figure 2. It is obvious that as the Rayleigh numbers increase, the differences in the profiles provided in Figure 2 are getting larger. These profiles are also comparable with a similar one in $[5,25]$. Combining with Table 4 , we can see that the two-grid variational multiscale Oseen iterative method is in good agreement with [5, 8, 24, 25].

Finally, we show the isotherms and streamlines of natural convection problem with the two-grid variational multiscale Oseen iterative scheme (4.1) at different Rayleigh numbers. We present these patterns in Figures 3 and 4. It is clear from the streamline pattern that, as the Rayleigh number increases, the circular vortex at the cavity center begins to deform into an ellipse and then breaks up into two vortices tending to approach to the corners of differentially heated sides of the cavity. Therefore, we can conclude that the flow is faster 
Table 4 Comparisons of maximum velocity at $y=0.5$ and $x=0.5$ with different methods $\left(h=\frac{1}{64}, H=\frac{1}{8}\right)$

\begin{tabular}{lccccccc}
\hline & SGFEM & OGVMM & TGVMM & Ref. [8] & Ref. [5] & Ref. [24] & Ref. [25] \\
\hline $\mathrm{Ra}=10^{3}$ & & & & & & & \\
$x=0.5$ & 3.6487 & 3.64869 & 3.64902 & 3.68 & - & 3.65 & 3.489 \\
$y=0.5$ & 3.69729 & 3.69777 & 3.69732 & 3.73 & - & 3.70 & 3.686 \\
$\mathrm{Ra}=10^{4}$ & & & & & & & \\
$x=0.5$ & 16.18 & 16.1815 & 16.1928 & 16.10 & 15.90 & 16.18 & 16.122 \\
$y=0.5$ & 19.6244 & 19.6317 & 19.6381 & 19.90 & 19.91 & 19.51 & 19.79 \\
$\mathrm{Ra}=10^{5}$ & & & & & & & \\
$x=0.5$ & 34.7186 & 34.8201 & 34.7183 & 34.00 & 33.51 & 34.81 & 33.39 \\
$y=0.5$ & 68.4705 & 68.5433 & 68.5738 & 70.00 & 70.60 & 68.22 & 70.63 \\
\hline
\end{tabular}

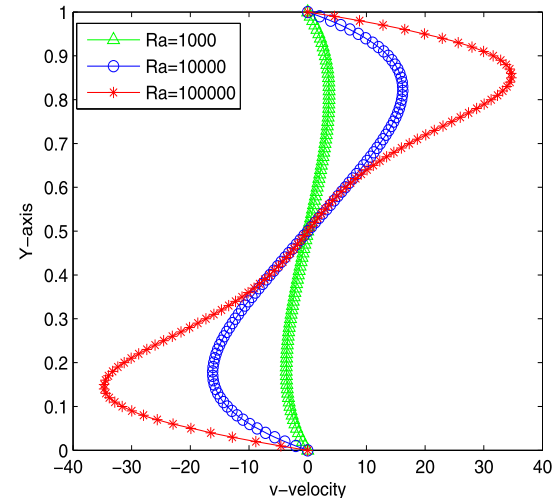

(a)

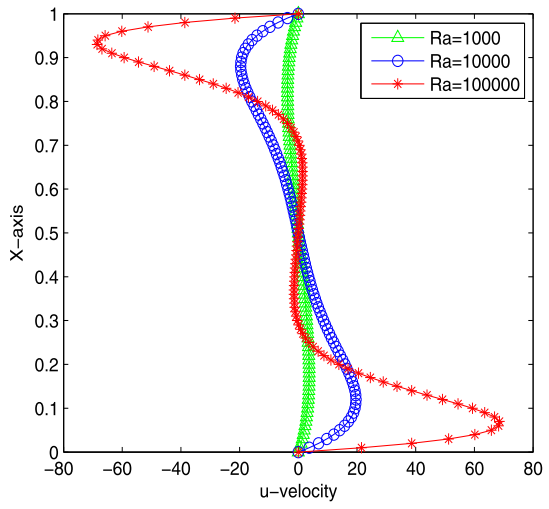

(b)

Figure 2 Comparison of velocity at the mid-width with different Rayleigh numbers. (a) vertical velocity, (b) horizontal velocity.

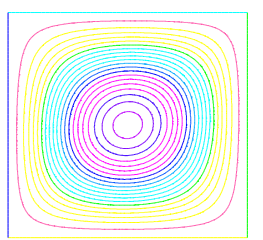

(a)

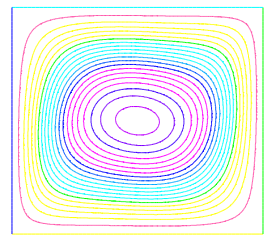

(b)

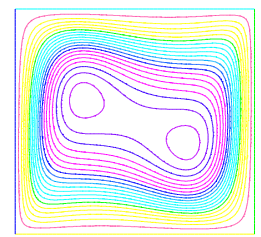

(c)

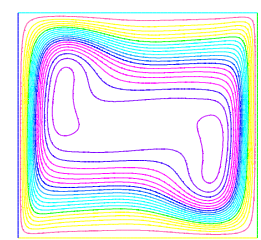

(d)

Figure 3 The streamlines of two level variational multiscale method for natural convection problem. (a) $R a=10^{3}$, (b) $R a=10^{4}$, (c) $R a=10^{5}$, (d) $R a=5 \times 10^{5}$.

as the thermal convection is concentrated. With the increase of Rayleigh numbers, the parallel behavior of temperature isolines is distorted as these lines seem to have a flat behavior in the central part of the region. Near the sides of the cavity, isolines tend to be vertical only. The temperature slopes with $\mathrm{Ra}=5 \times 10^{5}$ at the corners of the differentially heated sides are more immersed than the case of lower Rayleigh number. We note that these graphics are also perfectly comparable with the ones given in the investigations of $[5,8,24,25]$. Both the data and the graphics show the efficiency and effectiveness of the two-grid variational multiscale method. 


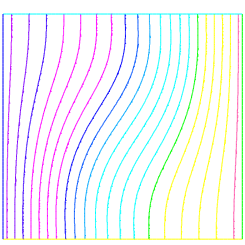

(a)

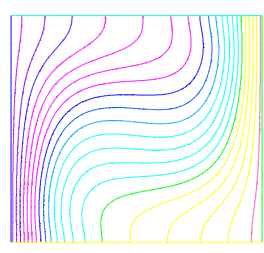

(b)

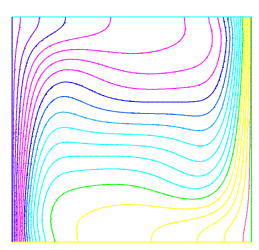

(c)

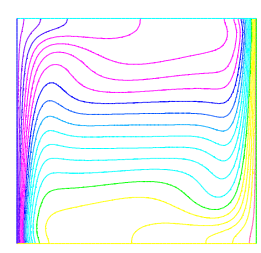

(d)

Figure 4 The isotherms of two level variational multiscale method for natural convection problem. (a) $R a=10^{3}$, (b) $R a=10^{4}$, (c) $R a=10^{5}$, (d) $R a=5 \times 10^{5}$.

\section{Competing interests}

The authors declare that no conflict of competing interests exists.

\section{Authors' contributions}

XSM carried out the main theorem and checked this paper, ZZT wrote this article. XSM, ZZT, and TZ read and approved the final manuscript.

\section{Author details}

${ }^{1}$ Center for Intelligence Science and Technology, School of Computer Science, Beijing University of Posts and Telecommunications, Beijing, 100876, P.R. China. ${ }^{2}$ School of Mathematics \& Information Science, Henan Polytechnic University, Jiaozuo, 454003, P.R. China. ${ }^{3}$ Departamento de Matemática, Centro Politécnico, Universidade Federal do Paraná, Curitiba, 81531-990, Brazil.

\section{Acknowledgements}

The authors are grateful to the editor and two referees for a number of helpful suggestions, which have greatly improved our original manuscript. This research is supported by CAPES and CNPq of Brazil (No. 88881.068004/2014.01), the NSF of China (No. 11301157), and the Foundation of Distinguished Young Scientists of Henan Polytechnic University (J2015-05)

Received: 20 December 2015 Accepted: 17 March 2016 Published online: 29 March 2016

\section{References}

1. Boland, J, Layton, W: An analysis of the finite element method for natural convection problems. Numer. Methods Partial Differ. Equ. 2, 115-126 (1990)

2. Gresho, PM, Lee, M, Chan, ST, Sani, RL: Solution of time dependent, incompressible Navier-Stokes and Boussinesq equations using the Galerkin finite element method. In: Approximation Methods for the Navier-Stokes Problems. Springer Lecture Notes in Mathematics, vol. 771, pp. 203-222. Springer, Berlin (1980)

3. Boland, J, Layton, W: Error analysis for finite element methods for steady natural convection problems. Numer. Funct. Anal. Optim. 11, 449-483 (1990)

4. Lenferink, HWJ: An accurate solution procedure for fluid flow with natural convection. Numer. Funct. Anal. Optim. 15, 661-687 (1994)

5. Cibik, A, Kaya, S: A projection based stabilized finite element method for steady-state natural convection problem. J. Math. Anal. Appl. 381, 469-484 (2011)

6. Galvin, KJ, Linke, A, Rebholz, LG, Wilson, NE: Stabilizing poor mass conservation in incompressible flow problems with large irrotational forcing and application to thermal convection. Comput. Methods Appl. Mech. Eng. 237-240, 166-176 (2012)

7. Zhang, T, Zhao, X, Huang, PZ: Decoupled two level finite element methods for the steady natural convection problem. Numer. Algorithms 68, 837-866 (2015)

8. Manzari, MT: An explicit finite element algorithm for convective heat transfer problems. Int. J. Numer. Methods Heat Fluid Flow 9, 860-877 (1999)

9. Benítez, M, Bermúdez, A: A second order characteristics finite element scheme for natural convection problems. J. Comput. Appl. Math. 235, 3270-3284 (2011)

10. Kaya, S, Layton, W, Riviere, B: Subgrid stabilized defect correction methods for the Navier-Stokes equations. SIAM J. Numer. Anal. 44, 1639-1654 (2006)

11. Guermond, JL, Marra, A, Quartapelle, L: Subgrid stabilized projection method for 2D unsteady flows at high Reynolds numbers. Comput. Methods Appl. Mech. Eng. 195, 5857-5876 (2006)

12. Layton, W: A connection between subgrid-scale eddy viscosity and mixed methods. Appl. Math. Comput. 133(1), 147-157 (2002)

13. Zhang, Y, He, YN: Assessment of subgrid-scale models for the incompressible Navier-Stokes equations. J. Comput. Appl. Math. 234, 593-604 (2010)

14. Galvin, KJ: New subgrid artificial viscosity Galerkin methods for the Navier-Stokes equations. Comput. Methods Appl. Mech. Eng. 200, 242-250 (2011)

15. Hughes, TJR: Multiscale phenomena: Green's functions the Dirichlet-to-Neumann formulation subgrid-scale models bubbles and the origins of stabilized methods. Comput. Methods Appl. Mech. Eng. 127, 387-401 (1995)

16. John, V, Kaya, S: A finite element variational multiscale method for the Navier-Stokes equations. SIAM J. Sci. Comput. 26, 1485-1503 (2005)

17. Kaya, S, Rivire, B: A two-grid stabilization method for solving the steady-state Navier-Stokes equations. Numer. Methods Partial Differ. Equ. 22, 728-743 (2005) 
18. Zheng, HB, Hou, YR, Shi, F, Song, LN: A finite element variational multiscale method for incompressible flows based on two local Gauss integrations. J. Comput. Phys. 228, 5961-5977 (2009)

19. Layton, W, Lee, H, Peterson, J: A defect-correction method for the incompressible Navier-Stokes equations. Appl. Math. Comput. 129, 1-19 (2002)

20. Liu, QF, Hou, YR: A two-level defect-correction method for Navier-Stokes equations. Bull. Aust. Math. Soc. 81, 442-454 (2010)

21. Zhang, YZ, Hou, YR, Zheng, HB: A finite element variational multiscale method for steady-state natural convection problem based on two local Gauss integrations. Numer. Methods Partial Differ. Equ. 30, 361-375 (2014)

22. Shang, YQ: A two-level subgrid stabilized Oseen iterative method for the steady Navier-Stokes equations. J. Comput. Phys. 233, 210-226 (2013)

23. Hecht, F, Pironneau, O, Hyaric, A, Ohtsuka, K: http://www.freefem.org/ff (2008)

24. de Vahl Davis, D: Natural convection of air in a square cavity: a benchmark solution. Int. J. Numer. Methods Fluids 3 249-264 (1983)

25. Wan, DC, Patnaik, BSV, Wei, GW: A new benchmark quality solution for the buoyancy driven cavity by discrete singular convolution. Numer. Heat Transf., Part B, Fundam. 40, 199-228 (2001)

Submit your manuscript to a SpringerOpen ${ }^{\circ}$ journal and benefit from:

- Convenient online submission

Rigorous peer review

- Immediate publication on acceptance

- Open access: articles freely available online

- High visibility within the field

- Retaining the copyright to your article 